\title{
Lecture on the Theory of Casimir phenomenae
}

\author{
Bart van Tiggelen
}

Laboratoire de Physique et Modélisation des Milieux Condensés Grenoble - France 


\section{Lecture on the Theory of Casimir phenomenae OED CNRS School 2012, IESC Cargèse , April 2012}

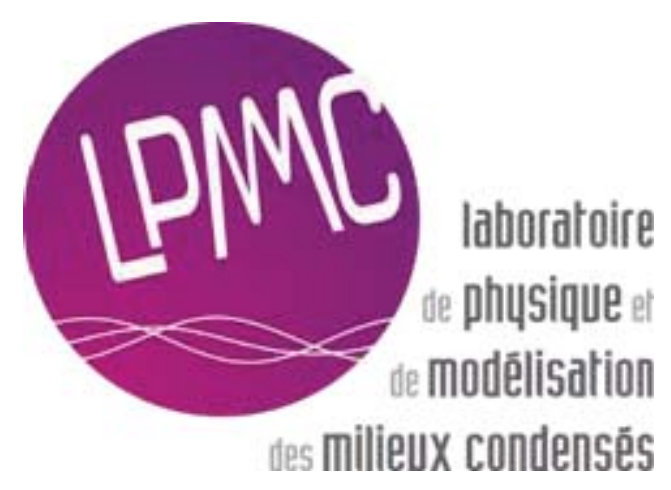

Bart van Tiggelen
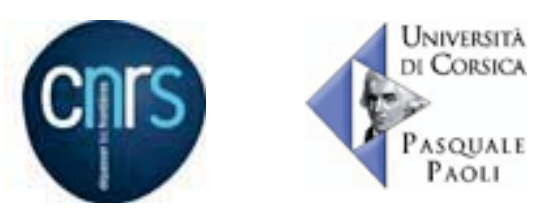


\section{Summary of course}

1. Important events

2. Lorentz invariance of Casimir energy

- Accelerated observer \&Unruh effect

- the UV catastrophe (Einstein equation,

sonoluminescence, (asimir momentum)

3. Fluctuation-dissipation theorem

4. Casimir force between ideal plates

- .. and on a metallic shell

- Proximity force approximation, dispersion, finite T)

4. Casimir-Polder attraction

5. Lifshitz formula

6. Quantum friction

7. Connection with QED:

- Casimir mass and Casimir momentum of $\mathrm{H}$.

8. Bibliography 


\subsection{Casimir energy}

1. Black body radiation (Planck, 1912 ', Einstein \& Stern, $1913^{2}$ )

$$
\frac{h v}{\exp (h v / k T)-1}+\frac{1}{2} h v=\left(k T-\frac{1}{2} h v\right)+\frac{1}{2} h v=k T+O(1 / T)
$$

2. Isotropic radiation with power spectrum $\omega^{3}$ is Lorentz-invariant

(Einstein, 1917³);

3. Van der Waals force $1 / t^{\circ}$ as a dispersion force due to quantum fluctuations (London, $1930^{4}$ )

3. Relation to Cosmological constant (Pauli, 1934, Davies, 19845)

4. Casimir Polder Force $1 / r^{7}\left(1948^{\circ}\right)$

5. Lamb shift (Lamb \& Retherford7, 1947, Bethe, 8 1947);

anomalous magnetic moment of electron (Schwinger ${ }^{19} 1948$ )

7. Attraction between metallic plates (Casimir, 194810),

refuted by Pauli as «absolute nonsense »

8. Lifshitz formula for dielectric bodies (Lifshitz, 1956 ${ }^{11}$ )

9. "The general theory of Van der Waals forces" (Lifshitz, Dzyalonishiniskii, Pitaevskii 1961'2) 


\subsection{Casimir energy}

10. Observation of Casimir effect (Sparnaay ${ }^{13}$ (100\%), 1958,

Lamoureux ${ }^{14}$ (5\%), 1997), Mohideen \& Roy ${ }^{15}, 1998$, Ederth $^{16}$ (1\%), 2000)

(the third for plane sphere-on cantilever geometry,

the latter for crossed cilinders)

11. Stability of the electron (Casimir, 1956 ${ }^{17}$, Boyer, 1968 ${ }^{18}$ )

12. Unruh effect \& Hawking radiation (Hawking 1974 19, Unruh 197620)

13. Dynamical Casimir effect (« moving mirror radiation $»)$, Fulling and Davies, $1976^{21}$

14. Bag model for hadrons (Jaffe etal, 197422)

15. Cosmological constant problem field theory (Weinberg, 198923)

16. Confined Casimir energy has inertial mass (Jeakel \&Reynaud, 199324) 


\subsection{Casimir energy}

17. Sonoluminescence as dynamical Casimir effect

(Schwinger, 1993225, Eberlein, 199626)

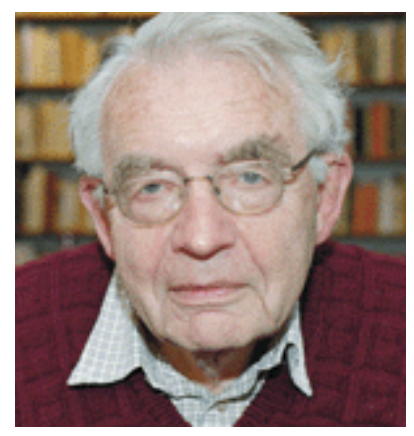

18. Quantum friction and shearing the quantum vacuum (Levitov, 198927;

Pendry, 199728)

19. Casimir dies at age of 90 (May 4, 2000)

20. Casimir momentum in magneto-electric media (Feigel, 200429)

21. An attractive Casimir force theorem for dielectrics (Kenneth, Klich, 2006 30)

22. Repulsive Van der Waals force in colloids (Feiler etal, 200831); quantum Casimir levitation of silicon sphere (Capasso etal 200932)

23. Casimir energy has gravitational mass (Milton, Fulling etal, 200733)

24. Scattering theory for Casimir energy: finite temperatures, beyond Proximity Force Approximation, corrugated surfaces

(Lambrecht \& Reynaud, Dalvit etal, Bordag etal34-37).

25. Observation of dynamical Casimir effect with SQVID (Wilson etal 2011)38

26. Observation of thermal Casimir force between plates, favoring Drude model (Sushkov, Dalvit, Lamoreaux, 2011) 39 


\subsection{Casimir energy before Casimir}

"At this point it should be noted that it is more consistent here, in contrast to the material oscillator, not to introduce a zero-point energy of $1 / 2^{-} h \omega$ per degree of freedom. For, on the one hand, the latter would give rise to an infinitely large energy per unit volume due to the infinite number of degrees of freedom, on the other hand, it would be principally unobservable since nor can it be emitted, absorbed or scattered and hence, cannot be contained within walls and, as is evident from experience, neither does it produce any gravitational field."

Pauli, Die Allgemeinen Prinzipien der Wellenmechanik, in Handbuch der Physik 241 (1933) 
2.1 Casimir energy is Lorentz invariant (to be continued)

$$
\begin{gathered}
d I(\theta, \omega)=\rho(\omega, \theta) d \omega d \cos \theta=d\left(1 / 2 \mathbf{E}^{2}+1 / 2 \mathbf{B}^{2}\right) \\
\boldsymbol{\beta}=\frac{\mathrm{v}}{c_{0}}(c \hat{\mathbf{k}}+s \cos \phi \hat{\mathbf{x}}+s \sin \phi \hat{\mathbf{y}})
\end{gathered}
$$

$$
\begin{array}{r}
\begin{array}{c}
\omega^{\prime}=\gamma \omega(1-\beta c) \\
c^{\prime}=\frac{c-\beta}{1-\beta c}
\end{array} \\
\frac{d\left(\omega^{\prime}, c^{\prime}\right)}{d(\omega, c)}=\frac{1}{\gamma(1-\beta c)} \\
\rho^{\prime}\left(\omega^{\prime}, c^{\prime}\right)=\rho\left(\frac{\omega^{\prime}}{\gamma(1-\beta c)}, c\right) \gamma^{3}(1-\beta c)^{3} \\
\rho(\omega, c)=\omega^{3} \text { Lorentz invariant (Einstein, } 1917 \text { 3) }
\end{array}
$$




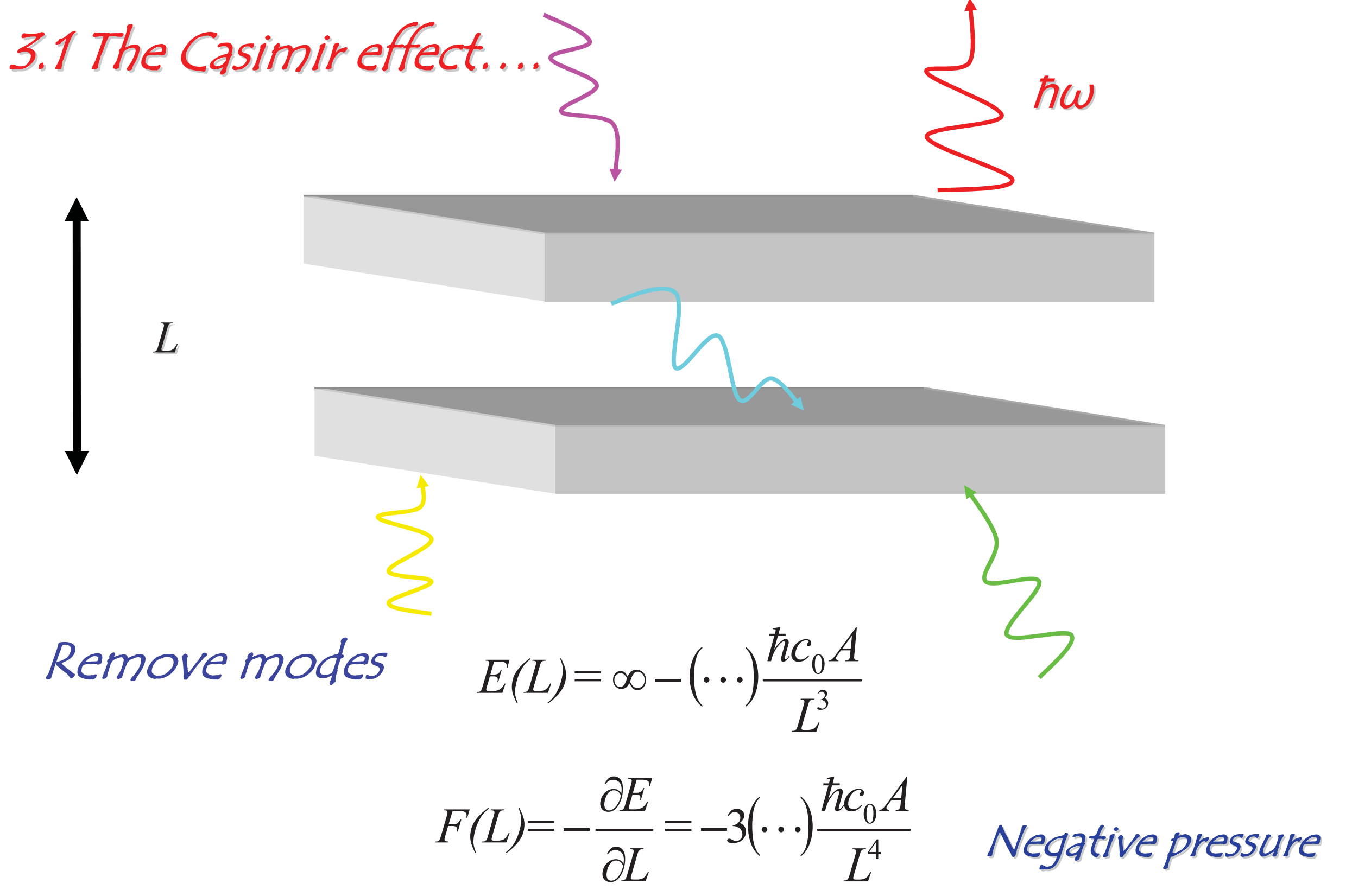

No momentum exchange between matter and radiation 


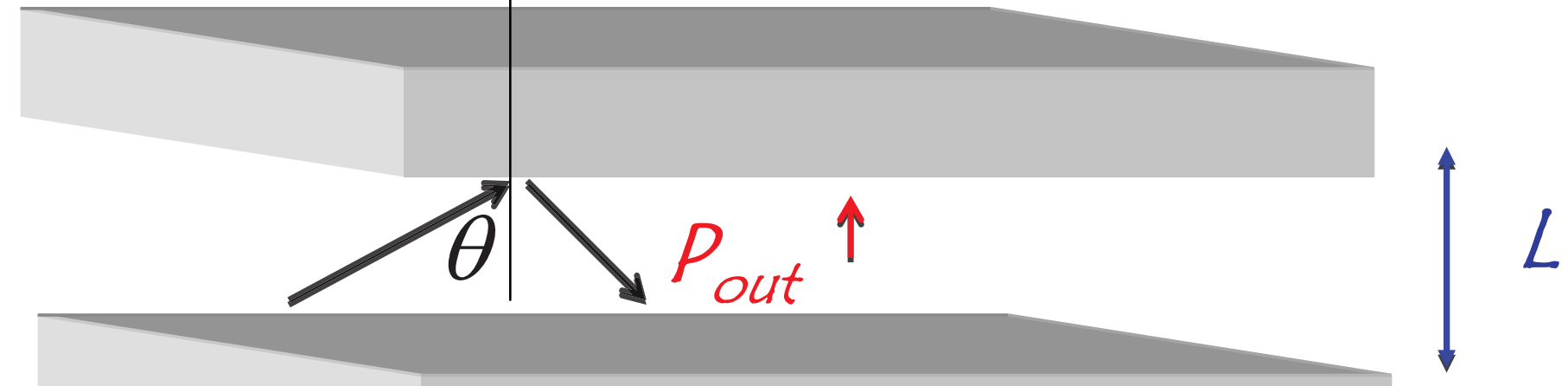

$P_{\text {out }}(\theta)=\frac{F\left(\Delta t=2 L / \cos \theta c_{0}\right)}{A}=\frac{2 \times 1 / 2 \hbar k \times \cos \theta}{A} \times \frac{c_{0} \cos \theta}{2 L} \times 2 \Rightarrow P_{\text {out }}=\frac{\hbar c_{0}}{V} \sum_{\substack{\text { modes } \\ \mathrm{k}_{z}>0}} \frac{k_{z}^{2}}{k}$

$$
\begin{aligned}
P=P_{\text {in }}-P_{\text {out }}=\frac{\hbar c_{0}}{L} \sum_{n=1}^{\infty} & \int \frac{d^{2} \mathbf{k}}{(2 \pi)^{2}} \frac{(n \pi / L)^{2}}{\sqrt{k^{2}+(n \pi / L)^{2}}} \\
& -\frac{\hbar c_{0}}{\pi} \int_{0}^{\infty} d k_{z} \int \frac{d^{2} \mathbf{k}}{(2 \pi)^{2}} \frac{k_{z}^{2}}{\sqrt{k^{2}+k_{z}^{2}}}
\end{aligned}
$$


3.3 The Casimir effect....

$$
\begin{aligned}
& F(n)=n^{2} \int_{0}^{\infty} d x \frac{1}{\sqrt{x+n^{2}}}=n^{2} \int_{n^{2}}^{\infty} \frac{d y}{\sqrt{y}} \\
& P=\frac{\hbar c_{0}}{4 \pi L}\left(\frac{\pi}{L}\right)^{3}\left[\sum_{n=1}^{\infty} F(n)-\int_{0}^{\infty} d n F(n)\right] \\
& =\frac{\pi^{2} \hbar c_{0}}{4 L^{4}}\left[-1 / 2 F(0)-1 / 12 F^{\prime}(0)+1 / 720 F_{\uparrow}^{\prime \prime \prime}(0)+\ldots\right] \\
& \begin{array}{lll}
0 & 0 & -12
\end{array} \\
& =-\frac{\pi^{2}}{240} \frac{\hbar c_{0}}{L^{4}} \quad 130 \mathrm{nN} / \mathrm{cm}^{2} / L^{4}(\mu \mathrm{m})
\end{aligned}
$$

Casimir energy diverges in UV but..

THE Casimir effect is a low energy phenomenon

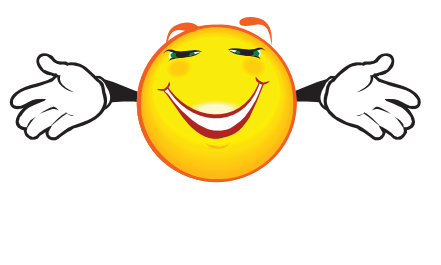


3.4 The Casimir effect of scalar bosons in $1 D$

$$
\begin{aligned}
F=F_{\text {in }}-F_{\text {out }} & =\frac{\hbar c_{0}}{2 L} \sum_{n=1}^{\infty} 2 \times \frac{1}{2} \frac{n \pi}{L}-\frac{\hbar c_{0}}{2 \pi} \int_{0}^{\infty} d k k \\
& =\frac{\hbar c_{0} \pi}{2 L^{2}} \lim _{\kappa \downarrow t 0}\left(\sum_{n=1}^{\infty} n \exp (-\kappa n)-\int_{0}^{\infty} d n n \exp (-\kappa n)\right) \\
& =\frac{-\pi}{24} \frac{\hbar c_{0}}{L^{2}}
\end{aligned}
$$


Proc. Koninklijke Nederlandse Academie voor Wetenschappen 51 (1948), 7910

(thank you Astrid Lambrecht for providing)

In order to obtain a finite result it is necessary to multiply the integrands by a function $\left[\left(k / k_{a}\right)\right.$ which is unity for $k\left\langle\left\langle k_{m}\right.\right.$ but tends to zero sufficiently rapidly for $\left(k / k_{m}\right) \rightarrow \infty$, where $k_{m}$ may be defined by $(1)=1$. The physical meaning is obvious: for very short waves ( $X$-rays e.g.) our plate is hardly an obstacle at all and therefore the zero point energy of these waves will not be influenced by the position of this plate.

Introduaino in il

We are thus led to the following conclusions. There exists an attractive force between two metal plates whtch is independent of the material of the plates as long as the distance is so large that for wave lengths comparable with that distance the penetration depth is small compored with the distance. This force may be interpreted as a zero point pressure of electromagnetic waves.

Although the effect is small, an experimental confirmation seems not unfeasable and might be of a certain interest. 
3.5 The Casimir effect at finite temperatures....

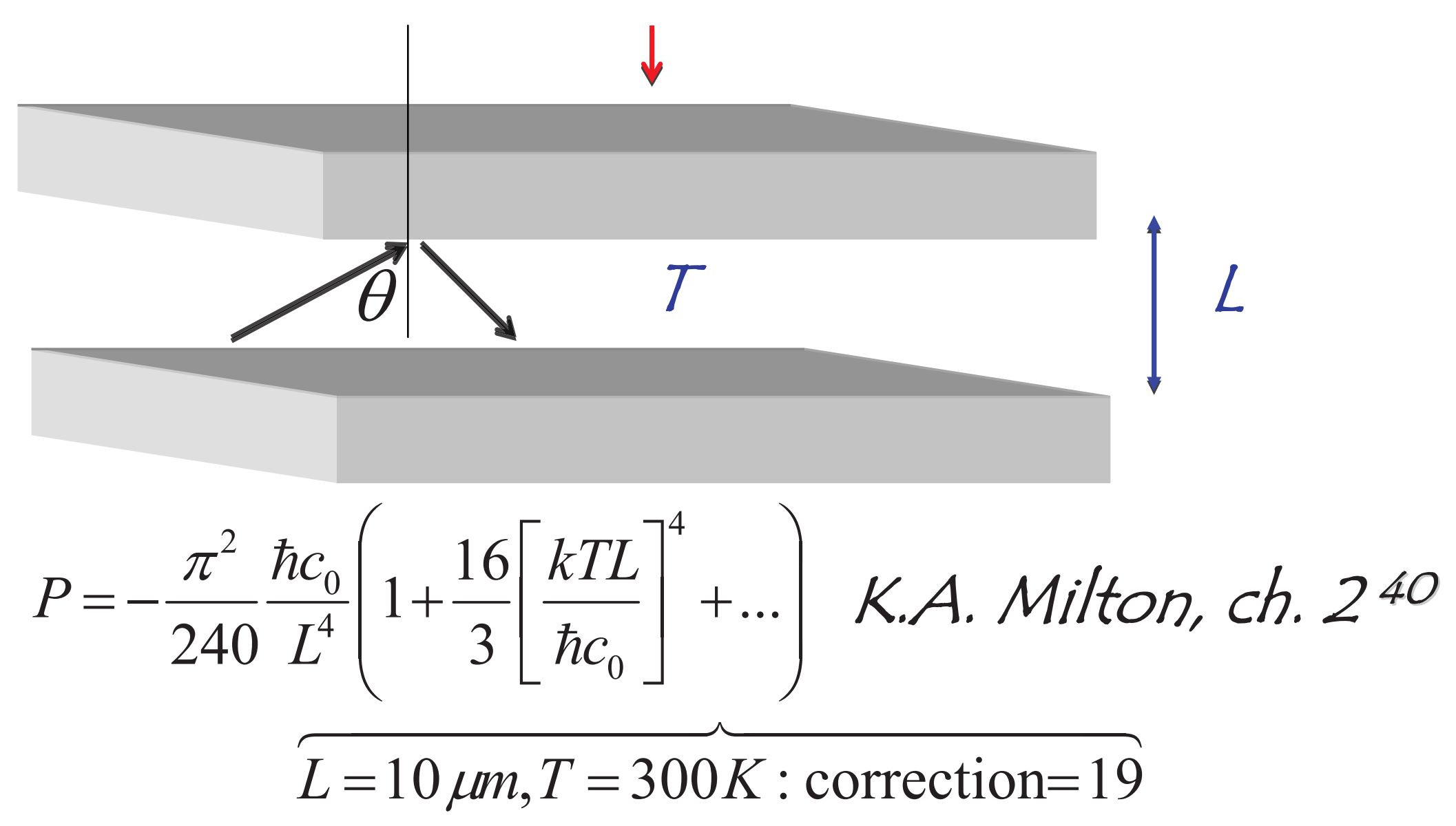

$\frac{k T L}{\hbar c_{0}}>>1: P=-2.4 \frac{k T}{4 \pi L^{3}}$ 


\subsection{The Casimir effect for a shell}

Does Casimir force stabilize the electron?

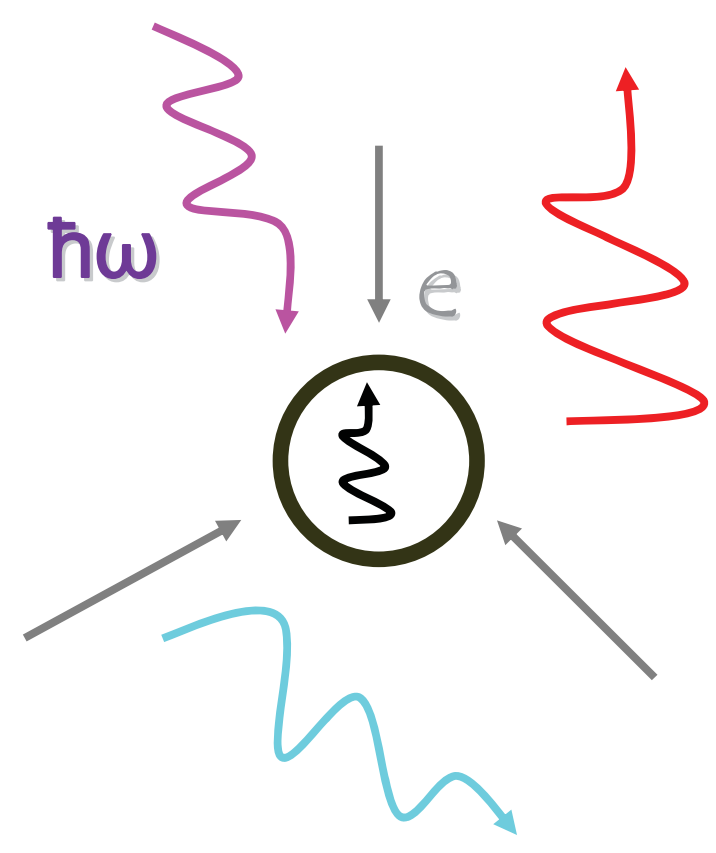

But force is repulsive!

Boyer (1968) 18

$$
F_{\text {Casimir }}=+0.094 \frac{\hbar c_{0}}{2 r_{e}^{2}}
$$

$$
\alpha=\frac{e^{2}}{4 \pi \varepsilon_{0} \hbar c_{0}}=0.0073 .
$$


4.1 Fluctuation dissipation theorem at finite temperature

$$
\begin{aligned}
& \begin{array}{c}
\rho=\frac{1}{Z} \exp (-\beta H) \quad H=\sum_{k} \hbar \omega_{k}\left(a_{k g}^{*} a_{k g}+1 / 2\right) \\
f_{k}=\frac{1}{\exp \left(\beta \hbar \omega_{k}\right)-1}
\end{array}\left\{\begin{array}{c}
\operatorname{Tr} \rho a_{k g}^{*} a_{k^{\prime} g^{\prime}}=f_{k} \delta_{k k^{\prime}} \delta_{g g^{\prime}} \\
\operatorname{Tr} \rho a_{k^{\prime} g^{\prime}} a_{k g}^{*}=\left(f_{k}+1\right) \delta_{k k^{\prime}} \delta_{g g^{\prime}}
\end{array}\right. \\
& \text { vacuum }
\end{aligned}
$$

$$
\mathbf{E}(\mathbf{r}, t)=\sum_{k g} i\left(\frac{\hbar \omega_{k}}{2 \varepsilon_{0}}\right)^{1 / 2}\left(a_{k^{\prime} g^{\prime}} \mathbf{E}_{k g}(\mathbf{r}) \exp \left(-i \omega_{k} t\right)-a_{k g}^{*} \mathbf{E}_{k g}^{*}(\mathbf{r}) \exp \left(i \omega_{k} t\right)\right)
$$$$
\mathbf{E}(\omega, \mathbf{r})=\int_{-\infty}^{+\infty} d t \mathbf{E}(t, \mathbf{r}) \exp (i \omega t) \quad \mathbf{E}(\omega)^{*}=\mathbf{E}(-\omega)
$$ 
4.2Fluctuation dissipation theorem at finite temperature

$$
\left.G^{ \pm}\left(\omega, \mathbf{r}, \mathbf{r}^{\prime}\right)\right)=\sum_{k g} \frac{\mathbf{E}_{k g}(\mathbf{r}) \mathbf{E}_{k g}^{*}\left(\mathbf{r}^{\prime}\right)}{(\omega \pm i 0)^{2}-\omega_{k}^{2}} \quad \begin{gathered}
\text { Classical retarded/advanced } \\
\text { Green function } \\
\text { for electric field }
\end{gathered}
$$

$$
\begin{array}{cl}
\omega>0: & \left\langle\mathrm{E}_{\mathrm{n}}(\mathbf{r}, \omega) \mathrm{E}_{\mathrm{m}}\left(\mathbf{r}^{\prime}, \omega^{\prime}\right)\right\rangle=2 \pi \delta_{\omega \omega^{\prime}} \frac{\hbar \omega^{2}}{2 \pi \varepsilon_{0}} i\left[G_{n m}^{+}\left(\omega, \mathbf{r}, \mathbf{r}^{\prime}\right)-G_{n m}^{-}\left(\omega, \mathbf{r}, \mathbf{r}^{\prime}\right)\right]\left(f_{\omega}+1\right) \\
& \left\langle\mathrm{E}_{\mathrm{n}}(\mathbf{r},-\omega) \mathrm{E}_{\mathrm{m}}\left(\mathbf{r}^{\prime},-\omega^{\prime}\right)\right\rangle=2 \pi \delta_{\omega \omega^{\prime}} \frac{\hbar \omega^{2}}{2 \pi \varepsilon_{0}} i\left[G_{n m}^{+}\left(\omega, \mathbf{r}, \mathbf{r}^{\prime}\right)-G_{n m}^{-}\left(\omega, \mathbf{r}, \mathbf{r}^{\prime}\right)\right] f_{\omega}
\end{array}
$$

No negative frequencies at $T=0$

$$
\left\langle\mathrm{E}_{\mathrm{n}}(\mathbf{r}, \omega) \mathrm{E}_{\mathrm{m}}\left(\mathbf{r}^{\prime}, \omega^{\prime}\right)+\mathrm{E}_{\mathrm{n}}(\mathbf{r},-\omega) \mathrm{E}_{\mathrm{m}}\left(\mathbf{r}^{\prime},-\omega^{\prime}\right)\right\rangle=2 \pi \delta_{\omega \omega^{\prime}} \frac{-\hbar \omega^{2}}{\pi \varepsilon_{0}} \operatorname{Im} G_{n m}^{+}\left(\omega, \mathbf{r}, \mathbf{r}^{\prime}\right)\left(2 f_{\omega}+1\right)
$$

$G^{-}(\omega)=G^{+}(-\omega) ; \mathbf{E}_{k g}(\mathbf{r}) \in \mathfrak{R}$ 
4.3 Fluctuation dissipation theorem at finite temperature

What if medium is dissipative (finite conductivity)?

$$
\left[\varepsilon_{0}+\frac{4 \pi i \sigma(\mathbf{r})}{\omega}\right] \omega^{2} \mathbf{E}(\mathbf{r}, \omega)+\nabla \times \nabla \times \mathbf{E}(\mathbf{r}, \omega)=\mathbf{j}(\mathbf{r}, \omega)
$$

dissipation

$$
\sigma>0
$$

\section{Current fuctuations}

$$
\begin{aligned}
& \sigma>0\left\langle j_{i}(\mathbf{r}, \omega) j_{j}^{*}\left(\mathbf{r}^{\prime}, \omega^{\prime}\right)\right\rangle=K \times 4 \pi \omega \sigma(\mathbf{r}) \times 2 \pi \delta_{\omega \omega^{\prime}} \delta_{i j} \delta_{\mathbf{r r}} \\
& \mathbf{E}(\mathbf{r}, \omega)=\int d \mathbf{x} G^{+}(\mathbf{r}, \mathbf{x}, \omega) \cdot \mathbf{j}(\mathbf{r}, \omega) \\
& \begin{aligned}
\Rightarrow\left\langle E_{n}(\mathbf{r}, \omega) E_{m}^{*}\left(\mathbf{r}^{\prime}, \omega^{\prime}\right)\right\rangle= & \int d \mathbf{x} \int d \mathbf{x}^{\prime} G_{n i}^{+}(\mathbf{r}, \mathbf{x}, \omega)\left\langle j_{i}(\mathbf{r}, \omega) j_{j}\left(\mathbf{r}^{\prime}, \omega^{\prime}\right)\right\rangle \cdot G_{j m}^{-}(\mathbf{x}, \mathbf{r}, \omega) \\
& =2 \pi \delta_{\omega \omega^{\prime}} K\left\langle\mathbf{r}\left|G^{+}(\omega) 4 \pi \omega \sigma(\mathbf{x}) G^{-}(\omega)\right| \mathbf{r}^{\prime}\right\rangle \\
& =2 \pi \delta_{\omega \omega^{\prime}} \frac{K}{-2 i}\left\langle\mathbf{r}\left|G^{+}(\omega)-G^{-}(\omega)\right| \mathbf{r}^{\prime}\right\rangle
\end{aligned}
\end{aligned}
$$

$K=\frac{\hbar \omega^{2}}{\pi \varepsilon_{0}} \operatorname{cotanh}(\beta \hbar \omega / 2)$

$$
\left\langle\mathrm{E}_{\mathrm{n}}(\mathbf{r}, \omega) \mathrm{E}_{\mathrm{m}}^{*}\left(\mathbf{r}^{\prime}, \omega^{\prime}\right)\right\rangle=2 \pi \delta_{\omega \omega^{\prime}} \frac{-\hbar \omega^{2}}{\pi \varepsilon_{0}} \operatorname{Im} G_{n m}^{+}\left(\omega, \mathbf{r}, \mathbf{r}^{\prime}\right)\left(2 f_{\omega}+1\right)
$$

Positive and negative frequencies added 


\subsection{Fluctuation dissipation theorem at finite temperature}

What if medium is dispersive and dissipative?

$$
\left[\varepsilon(\mathbf{r})+\frac{4 \pi i \sigma(\mathbf{r})}{\omega}\right] \omega^{2} \mathbf{E}(\mathbf{r}, \omega)+\nabla \times \nabla \times \mathbf{E}(\mathbf{r}, \omega)=\mathbf{j}(\mathbf{r}, \omega)
$$

dispersion dissipation

$$
\sigma>0
$$

current fluctuations

Substitute $\quad \boldsymbol{\psi}(\mathbf{r}, \omega)=\sqrt{\varepsilon(\mathbf{r})} \mathbf{E}(\mathbf{r}, \omega)$ and show that

$$
\left\langle\mathrm{E}_{\mathrm{n}}(\mathbf{r}, \omega) \mathrm{E}_{\mathrm{m}}^{*}\left(\mathbf{r}^{\prime}, \omega^{\prime}\right)\right\rangle=2 \pi \delta_{\omega \omega^{\prime}} \frac{-\hbar \omega^{2}}{\pi \sqrt{\varepsilon(\mathbf{r}) \varepsilon\left(\mathbf{r}^{\prime}\right)}} \operatorname{Im} G_{n m}^{+}\left(\omega, \mathbf{r}, \mathbf{r}^{\prime}\right)\left(2 f_{\omega}+1\right)
$$

Dangerous to quantize macroscopic media! 
2. 2 continued: Casimir energy is Lorentz invariant

$$
\begin{aligned}
& \left.\begin{array}{c}
\partial_{t} \frac{1}{2}\left(\varepsilon_{0} E^{2}+\frac{1}{\mu_{0}} B^{2}+c_{0}^{2} \nabla \cdot \varepsilon_{0} \mathbf{E} \times \mathbf{B}\right. \\
\partial_{t}\left(\varepsilon_{0} \mathbf{E} \times \mathbf{B}\right)+\nabla \cdot\left\{\frac{1}{2}\left(\varepsilon_{0} E^{2}+\frac{1}{\mu_{0}} B^{2}\right) \delta_{i j}-\left(\varepsilon_{0} E_{i} E_{j}+\frac{1}{\mu_{0}} B_{i} B_{j}\right)\right\}=0
\end{array}\right]\left[\begin{array}{c}
\partial_{\mu} T^{\mu}{ }_{\nu}=0 \\
T^{\mu}{ }_{\mu}=0 \\
T_{\mu \nu}=T_{\nu \mu}
\end{array}\right. \\
& \frac{\omega^{2}}{c_{0}^{2}} G_{i j}(\omega, \mathbf{k})=\left(\frac{\omega^{2}}{c_{0}^{2}} \delta_{i j}-k_{i} k_{j}\right) \frac{1}{(\omega+i 0)^{2} / c_{0}^{2}-k^{2}} \\
& \left\langle T_{\mu \nu}\right\rangle \propto-2 \hbar \operatorname{Im} \int \frac{d \omega}{2 \pi} \int \frac{d^{3} \mathbf{k}}{(2 \pi)^{3}} \frac{1}{\frac{(\omega+i 0)^{2}}{c_{0}^{2}}-k^{2}}\left(\begin{array}{cc}
\omega^{2} / c_{0}^{2} & \omega k_{j} / c_{0} \\
\omega k_{i} / c_{0} & k_{i} k_{j}
\end{array}\right)
\end{aligned}
$$


2.3 continued: Casimir energy is Lorentz invariant

$$
\begin{gathered}
\operatorname{Im} \int_{0}^{\infty} \frac{d \omega}{2 \pi} \frac{\omega^{2} / c_{0}^{2}}{(\omega+i 0)^{2}}-k^{2} \\
\operatorname{Im} \int_{0}^{\infty} \frac{d \omega}{2 \pi}\left[\frac{\omega^{2} / c_{0}^{2}}{\frac{(\omega+i 0)^{2}}{c_{0}^{2}}-k^{2}}-1\right]=\operatorname{Im} \int_{0}^{\infty} \frac{d \omega}{2 \pi} \frac{k^{2}}{\frac{(\omega+i 0)^{2}}{c_{0}^{2}}-k^{2}}=-\int_{0}^{\infty} d \zeta \frac{k^{2}}{\frac{\zeta^{2}}{c_{0}^{2}}+k^{2}} \\
\text { (Milton, chapter 10; 1997 40 }
\end{gathered}
$$

Not cosmological constant but ultrarelativistic dust $E=3 P$ ( $L \& L$, Theory of Fields) Divergence of Lorentz invariant theory is not Lorentz invariant? 


\section{4 continued Casimir energy is Lorentz invariant}

Wick rotation when regularized invariantly:

Analytic function

$\omega \rightarrow i \zeta c_{0}:\left\langle T_{\mu v}\right\rangle=\frac{\hbar c_{0}}{8 \pi^{4}} \int_{0}^{\infty} d \zeta \int d^{3} \mathbf{k} \frac{1}{\zeta^{2}+k^{2}}\left(\begin{array}{cc}-\zeta^{2} & i \zeta k_{i} \\ i \zeta k_{j} & k_{i} k_{j}\end{array}\right) \begin{gathered}\text { bounded in C } \\ f\left(\zeta^{2}+k^{2}\right)\end{gathered}$

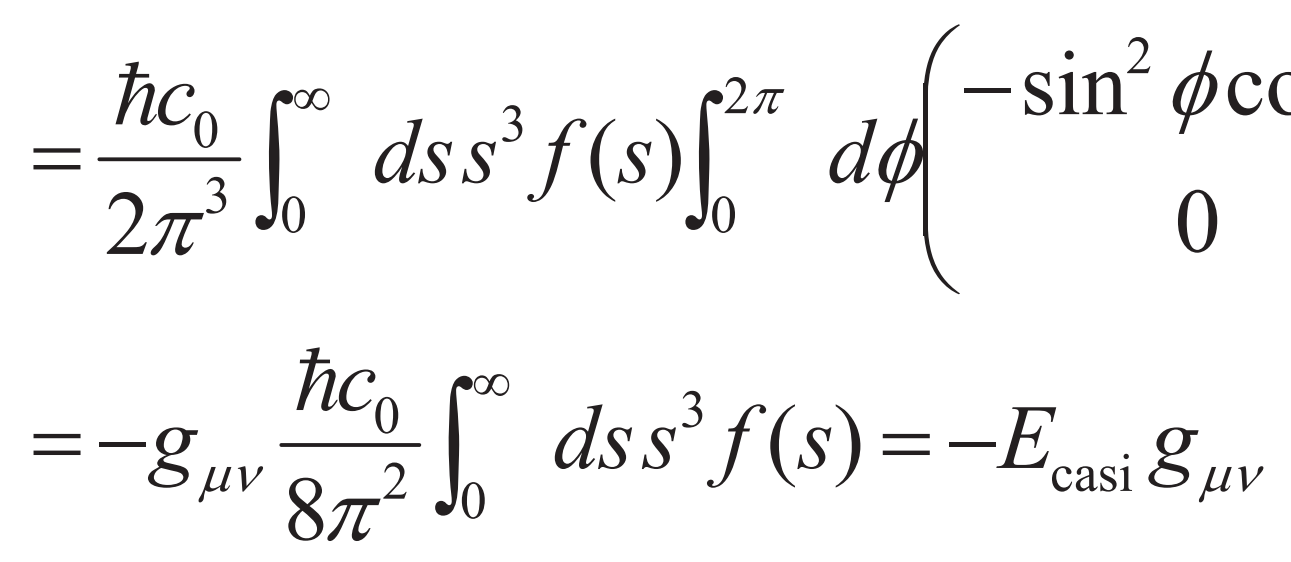

$1 / 3 \delta_{i j} \sin ^{4} \phi$

Regularized quantum vacuum is formal candidate for cosmological constant

$$
R_{\mu v}-R g_{\mu v}+\Lambda g_{\mu v}=-\frac{8 \pi G}{c_{0}^{4}} T_{\mu v}
$$$$
\Rightarrow \Lambda=\frac{8 \pi G}{c_{0}^{4}} E_{\text {casi }}
$$

$$
\frac{\Lambda c_{0}^{4}}{8 \pi G} \approx \rho_{c}=10^{-5} \mathrm{GeV} / \mathrm{cm}^{3}:=\frac{\hbar c}{8 \pi^{2}} \int_{0}^{1 / a} d s s^{3} \Rightarrow a \approx 13 \mu \mathrm{m}
$$

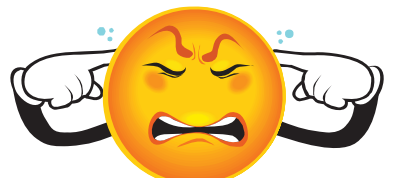

Is regularization result of stabilized extra, large « enrolled 》 dimensions d $>4$ of this size? 
2.5 Non Newtonian gravity deduced from Casimir experiments

$$
1 \mathrm{~mm} \text { thick plate }\left(10 \mathrm{~g} / \mathrm{cm}^{3}\right) \mathrm{F}_{\text {casi }}=\mathrm{F}_{\mathrm{z}} \rightarrow \mathrm{L}=13 \mu \mathrm{m}
$$



E. Adelberger et al (2003) 41

Kapner etal, $2007^{42}$ Sushkov, Kim, Dalvit, Lamoreaux ${ }^{43} 2011$

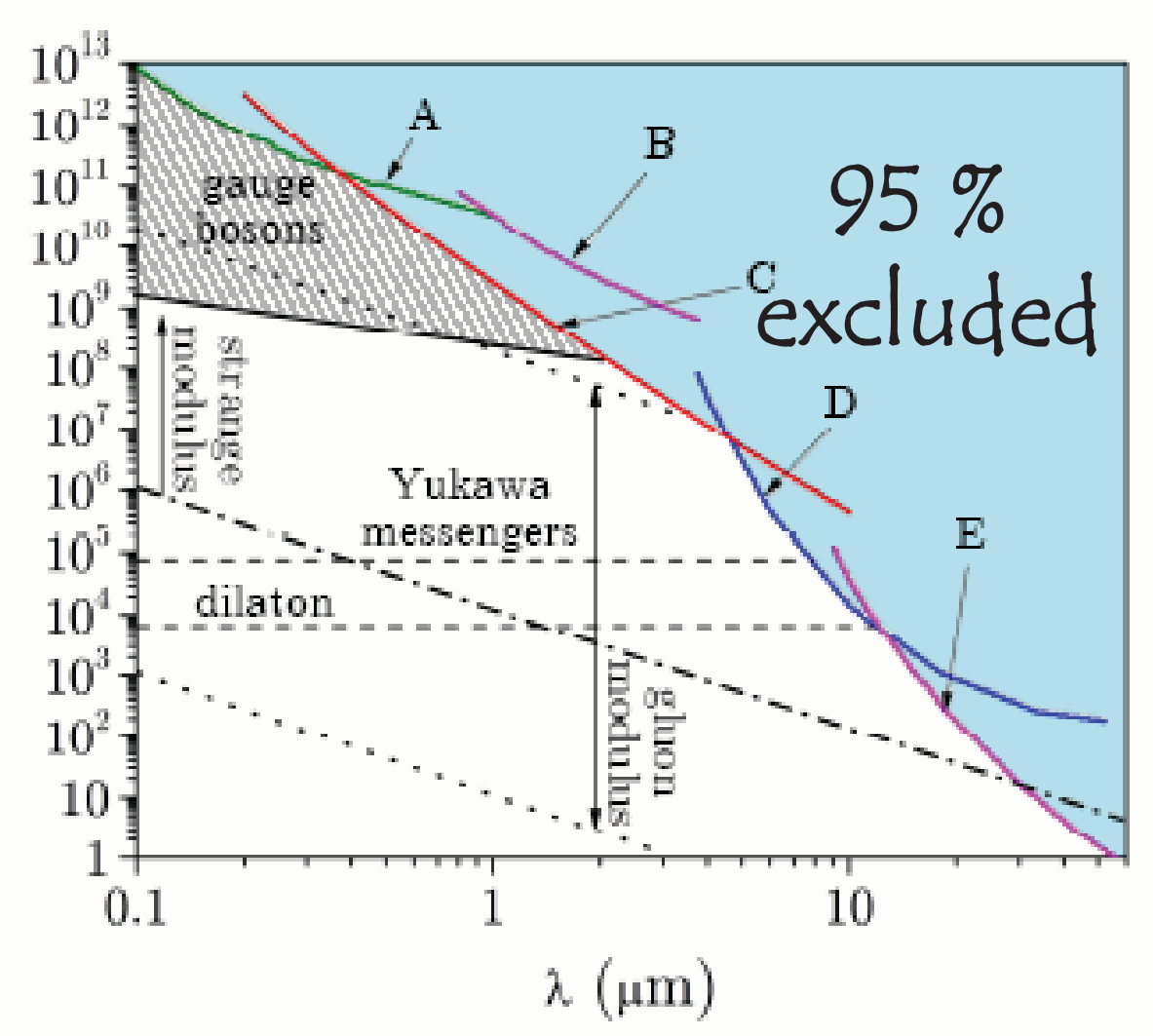




\subsection{Accelerated Casimir energy becomes Planck law}

Constant acceleration a in comoving frame with speed v (Milonni, 1994 44)
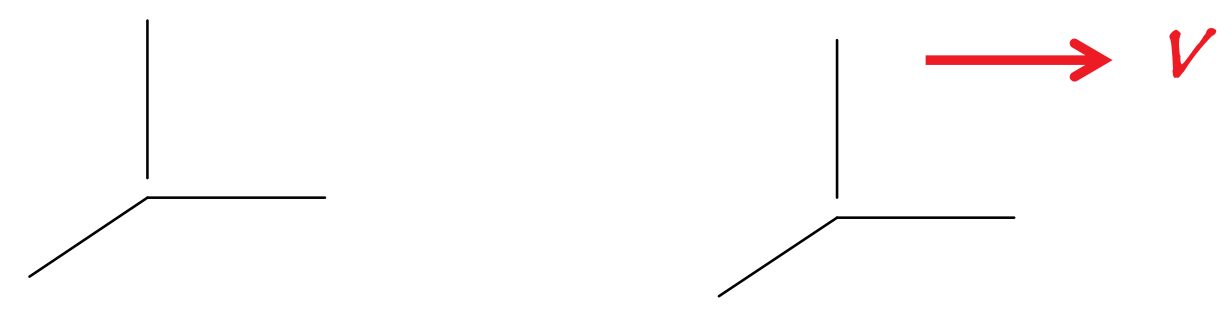

$$
\frac{d u_{/ /}{ }^{\prime}}{d t^{\prime}}=\frac{d u_{/ /}}{d t} \frac{1}{\gamma} \frac{1}{\left(1-\mathbf{v} \cdot \mathbf{u} / c_{0}^{2}\right)^{2}}=a \text { for } \mathbf{u}=\mathbf{v} \Rightarrow \frac{d v}{d t}=a\left(1-\frac{v^{2}}{c_{0}^{2}}\right)^{3 / 2}
$$

$$
\begin{gathered}
v(t)=\frac{a t}{\sqrt{1+a^{2} t^{2} / c_{0}^{2}}} \longrightarrow d \tau=\sqrt{1-v^{2} / c_{0}^{2}} d t \Rightarrow t(\tau)=\frac{c_{0}}{a} \sinh \frac{a \tau}{c_{0}} \\
x(t)=\frac{c_{0}^{2}}{a}\left[\sqrt{1+a^{2} t^{2} / c_{0}^{2}}-1\right] \\
\longrightarrow x(\tau)=\frac{c_{0}^{2}}{a}\left(\cosh \frac{a \tau}{c_{0}}-1\right) \\
x^{2}(\tau)-t^{2}(\tau)=-\frac{c_{0}^{4}}{a^{2}} \sinh ^{2} \frac{a \tau}{2 c_{0}}
\end{gathered}
$$




\subsection{Accelerated Casimir energy becomes Planck law}

$$
\begin{aligned}
& \mathbf{E}=\partial_{t} \mathbf{A} \quad\left\langle\mathrm{A}_{\mathrm{n}}(\mathbf{r}, \omega) \mathrm{A}_{\mathrm{m}}^{*}\left(\mathbf{r}^{\prime}, \omega\right)\right\rangle=2 \pi \delta_{\omega \omega^{\prime}} \frac{-\hbar}{\pi \varepsilon_{0}} \operatorname{Im} G_{n m}^{+}\left(\omega, \mathbf{r}, \mathbf{r}^{\prime}\right)\left(2 f_{\omega}+1\right) \\
& T=0 ; a \neq 0:\left\langle\mathrm{A}_{\mathrm{n}}(0, t=0) \mathrm{A}_{\mathrm{m}}^{*}(\mathbf{r}(t), t)\right\rangle=\frac{\hbar}{\varepsilon_{0}} \int_{0}^{\infty} d \omega \int \frac{d^{3} \mathbf{k}}{(2 \pi)^{3}} \delta\left(\frac{\omega^{2}}{c_{0}^{2}}-k^{2}\right) \delta_{n m} \exp (i \mathbf{k r}(t)-i \omega t) \\
& =\frac{\hbar}{(2 \pi)^{3} c_{0}} \frac{\delta_{n m}}{r^{2}-c_{0}^{2} t^{2}} \propto-\frac{\hbar a^{2} / c_{0}^{5}}{\sinh ^{2} \frac{a \tau}{2 c_{0}}} \\
& T \neq 0 ; a=0:\left\langle\mathrm{A}_{\mathrm{n}}(0, t=0) \mathrm{A}_{\mathrm{m}}^{*}(\mathbf{r}=0, t)\right\rangle=\frac{\hbar}{\varepsilon_{0}} \int_{0}^{\infty} d \omega \int \frac{d^{3} \mathbf{k}}{(2 \pi)^{3}} \delta\left(\frac{\omega^{2}}{c_{0}^{2}}-k^{2}\right) \delta_{n m} \exp (-i \omega t)\left(2 f_{\omega}+1\right) \\
& =\ldots-\frac{\hbar(\pi k T / \hbar)^{2} / c_{0}^{3}}{\sinh ^{2}\left(\frac{\pi k T}{\hbar} \tau\right)} \\
& T=\frac{\hbar a}{2 \pi k c_{0}} \\
& \text { Unruh/Hawking temperature }
\end{aligned}
$$


4.1 Inertial Casimir mass?

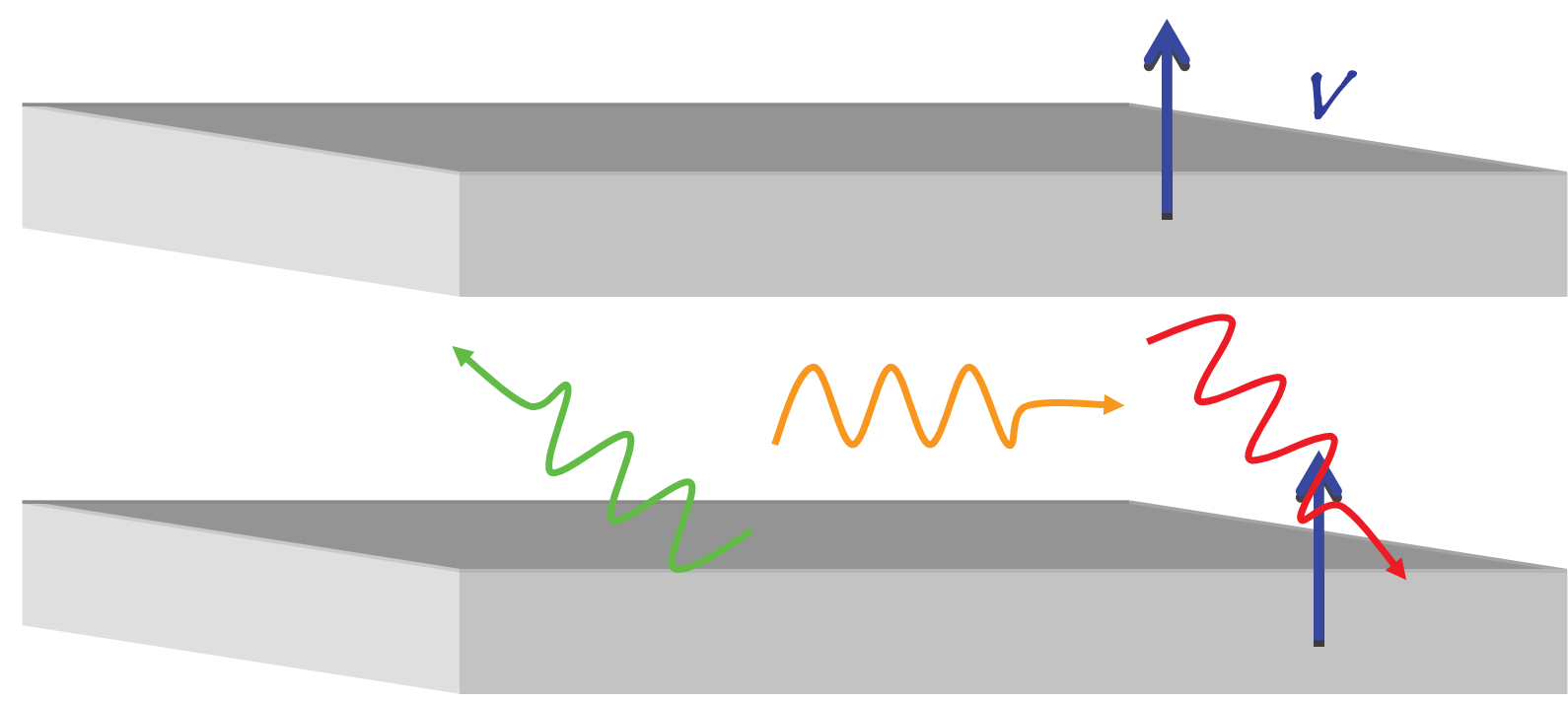

question

$$
\mathbf{P} / A=m \mathbf{v} / A=\frac{M+E_{\text {casi }} / A}{c_{0}^{2}} \mathbf{v}=\frac{M / A-\pi^{2} / 20 \hbar c_{0} / L^{2}}{c_{0}^{2}} \mathbf{v}
$$


4.2 Casimir friction?

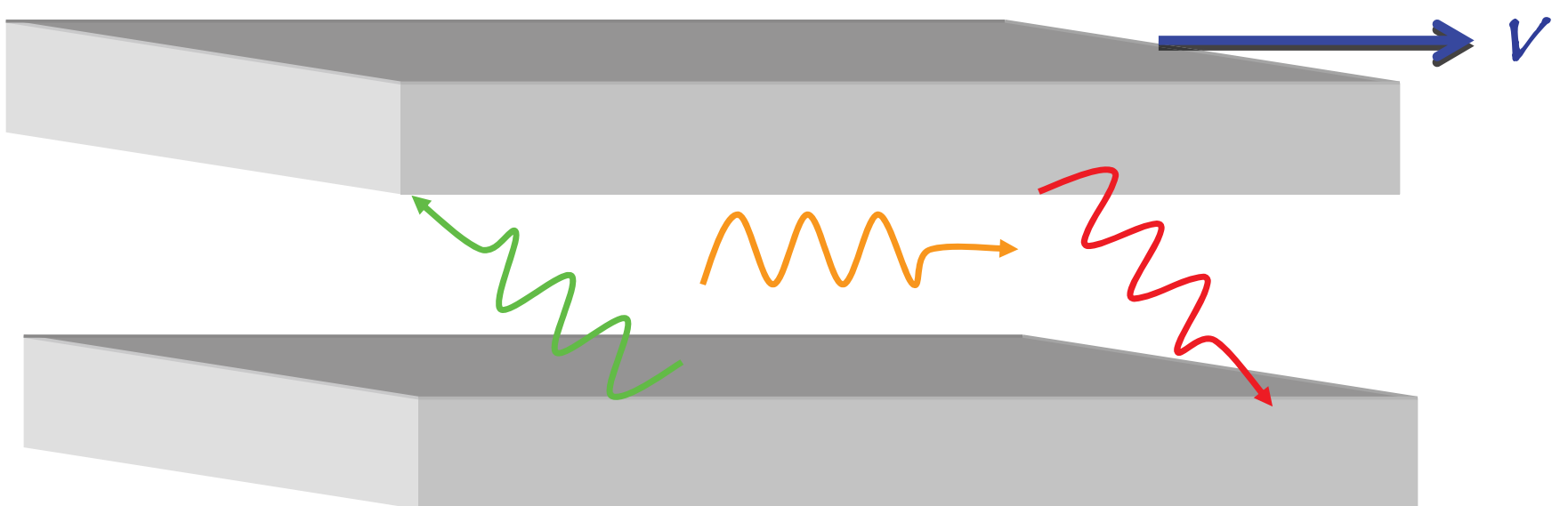

question

$$
\mathbf{F} / A=-\gamma \mathbf{v} \quad ?
$$




\section{3 vacuum force on moving object}

Fulling and Davies (1976 21)

$$
F(t)=\frac{\hbar}{6 \pi c_{0}^{2}} z^{\prime \prime \prime}(t)
$$

$F=O$ for uniform speed (by Lorentz invariance) or acceleration (Unruh effect)

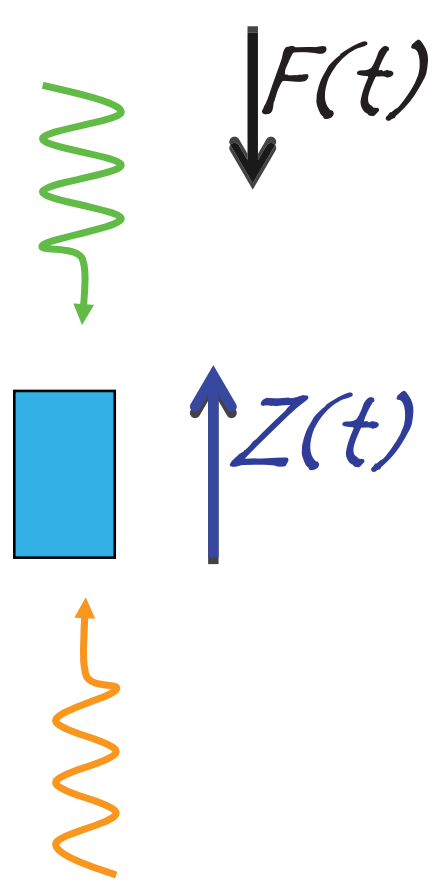




\section{4 vacuum force on moving mirrors: Casimir mass}

Jaekel and Reynaud(1993 24)

Retarded reaction force from mirrror 2 via vacuum

\section{$F(t)$}

$$
\begin{aligned}
\text { on mirror } 1 & \text { J } \\
\delta F_{1}(t)= & \frac{\hbar}{6 \pi c_{0}^{2}}\left(z_{1}^{\prime \prime}(t)-z_{2}^{\prime \prime \prime}(t-\tau)+z_{1}^{\prime \prime}(t-2 \tau) \mp \ldots\right) \\
& -\frac{\hbar c_{0} \pi \tau}{12 L^{3}}\left(z_{1}^{\prime}(t)-z_{2}^{\prime}(t-\tau)+z_{1}^{\prime}(t-2 \tau) \mp \ldots\right)
\end{aligned}
$$

Retarded variaton in Casimir force $\delta F_{1}(t)=-F^{\prime}\left(z_{2}-z_{1}\right) \times z_{1}{ }^{\prime}(t) \times \tau$

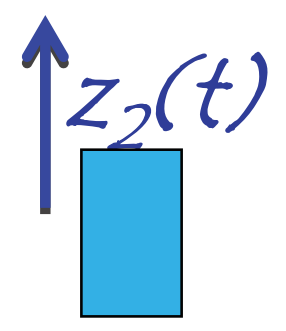

Keep $z_{1}-z_{2}$ constant: $z^{\prime}=z_{1}^{\prime}=z_{2}^{\prime}$
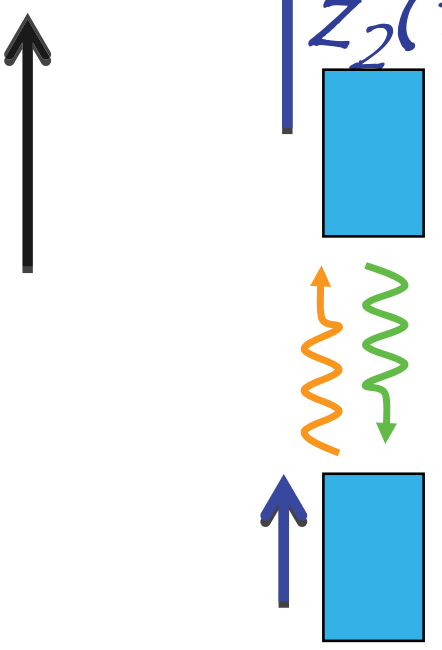

$z_{1}(t)$

$$
\begin{aligned}
& \delta F_{1+2}(\omega)=2\left(\frac{i \hbar \omega^{3}}{6 \pi c_{0}^{2}}+\frac{i \omega \hbar c_{0} \pi \tau}{12 L^{3}}\right)(1-\exp (i \omega \tau)+\exp (2 i \omega \tau) \mp \ldots) z(\omega) \\
& =\frac{\frac{i \hbar \omega}{6 \pi c_{0}^{2}}\left(\omega^{2}+\frac{\pi^{2}}{2 \tau^{2}}\right)}{\cos 1 / 2 \omega \tau} \exp (-1 / 2 i \omega \tau) z(\omega)=-\frac{\hbar \pi}{12 L^{2}}(-i \omega) z(\omega)-\frac{\hbar}{24 \pi c_{0} L}(-i \omega)^{2} z(\omega)
\end{aligned}
$$

Quantum friction (not found by ref 24)! $\quad \gamma=\frac{\hbar \pi}{L^{2}} \quad m=\frac{E_{\text {casi }}}{c_{0}^{2}}$ Casimir mass 
5.1 Van der Waals and Casimir Polder interaction

$$
\begin{gathered}
\left\langle\mathrm{E}_{\mathrm{n}}(\mathbf{r}, \omega) \mathrm{E}_{\mathrm{m}}^{*}\left(\mathbf{r}^{\prime}, \omega^{\prime}\right)\right\rangle=2 \pi \delta_{\omega \omega^{\prime}} \frac{-\hbar \omega^{2}}{\pi \sqrt{\varepsilon(\mathbf{r}) \varepsilon\left(\mathbf{r}^{\prime}\right)}} \operatorname{Im} G_{n m}^{+}\left(\omega, \mathbf{r}, \mathbf{r}^{\prime}\right)\left(2 f_{\omega}+1\right) \\
\left\langle\int d \mathbf{r}\left[\frac{1}{2} \varepsilon(\mathbf{r}) \mathbf{E}^{2}(\mathbf{r}, t)+\frac{1}{2 \mu_{0}} \mathbf{B}^{2}(\mathbf{r}, t)\right]\right\rangle=-2 \hbar \operatorname{Im} \int_{0}^{\infty} \frac{d \omega}{2 \pi} \int \frac{d \mathbf{k}}{(2 \pi)^{3}}\left(k^{2} \delta_{n m}-k_{n} k_{m}\right) G_{n m}^{+}(\omega, \mathbf{k}, \mathbf{k})\left(2 f_{\omega}+1\right) \\
T-\text { matrix of } 2 \text { point dipoles: } \\
\mathbf{T}_{\mathbf{k} \mathbf{k}^{\prime}}(\omega)=\frac{t(\omega) e^{i\left(\mathbf{k}-\mathbf{k}^{\prime}\right) \mathbf{r}_{1}}+t(\omega) e^{i\left(\mathbf{k}-\mathbf{k}^{\prime}\right) \mathbf{r}_{2}}+t^{2} \mathbf{G}_{0}^{+}\left(\omega, \mathbf{r}_{12}\right)\left[e^{i \mathbf{k} \mathbf{r}_{1}-i \mathbf{k}^{\prime} \mathbf{r}_{2}}+e^{i \mathbf{k r} \mathbf{r}_{2}-i \mathbf{k}^{\prime} \mathbf{r}_{1}}\right]}{1-t^{2} \mathbf{G}_{0}^{2}\left(\omega, \mathbf{r}_{12}\right)} \\
\mathbf{G}^{+}(\omega, \mathbf{k})=\frac{1-c_{0}^{2} \mathbf{k} \mathbf{k} / \omega^{2}}{(\omega+i 0)^{2} / c_{0}^{2}-k^{2}}
\end{gathered}
$$

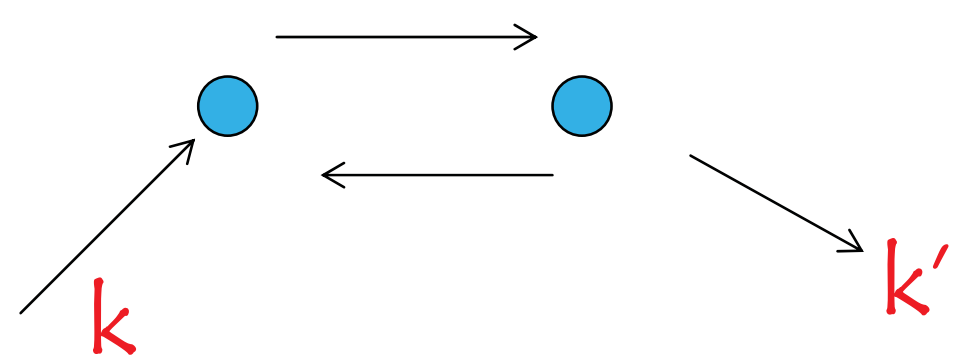




\subsection{Van der Waals interaction}

Van Tiggelen, 1999 45)

$\langle\delta E\rangle=-\hbar \operatorname{Im} \operatorname{Tr} \int_{0}^{\infty} \frac{d \omega}{2 \pi} \omega \frac{d}{d \omega}\left\{2 \log t(\omega)+\log \left(1-t^{2}(\omega) \mathbf{G}^{2}\left(\omega, \mathbf{r}_{12}\right)\right\}\right.$ $=\hbar \operatorname{Im} \operatorname{Tr} \int_{0}^{\infty} \frac{d \omega}{2 \pi} 2 \log t(\omega)+\hbar \operatorname{Im} \operatorname{Tr} \int_{0}^{\infty} \frac{d \omega}{2 \pi} \log \left(1-t^{2}(\omega) \mathbf{G}^{2}\left(\omega, \mathbf{r}_{12}\right)\right)$

$\langle\delta E\rangle=2 \times 3 / 2 \hbar \omega_{0}$

$$
r_{12}<\omega_{0} / c_{0}
$$

Ground state energy

$$
=
$$

Casimir energy

(this anticipates Lamb shift as a « (asimir effect 》)

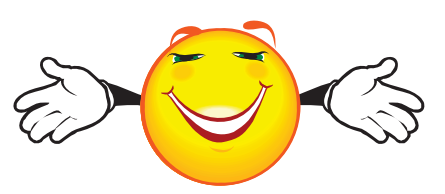

$$
\langle\delta E\rangle=\hbar \operatorname{Im} \operatorname{Tr} \int_{0}^{\infty} \frac{d \omega}{2 \pi}\left\{-t^{2}(\omega)\left(\frac{1-3 \hat{\mathbf{r}} \hat{\mathbf{r}}}{4 \pi \omega^{2} r^{3} / c_{0}^{2}}\right)^{2}\right\}
$$$$
=-6 \hbar \int_{0}^{\infty} \frac{d s}{2 \pi}\left(\frac{\alpha(i s)}{4 \pi}\right)^{2} \frac{1}{r^{6}}=-\frac{3}{4} \hbar \omega_{0} \frac{\alpha(0)^{2}}{(4 \pi)^{2} r^{6}}
$$

Van de Waals energy = Casimir energy 


\subsection{Casimir Polder interaction}

$\langle\delta E\rangle=-\hbar \operatorname{Im} \operatorname{Tr} \int_{0}^{\infty} \frac{d \omega}{2 \pi} \omega \frac{d}{d \omega}\left\{2 \log t(\omega)+\log \left(1-t^{2}(\omega) \mathbf{G}^{2}\left(\omega, \mathbf{r}_{12}\right)\right\}\right.$ $=\hbar \operatorname{Im} \operatorname{Tr} \int_{0}^{\infty} \frac{d \omega}{2 \pi} 2 \log t(\omega)+\hbar \operatorname{Im} \operatorname{Tr} \int_{0}^{\infty} \frac{d \omega}{2 \pi} \log \left(1-t^{2}(\omega) \mathbf{G}^{2}\left(\omega, \mathbf{r}_{12}\right)\right.$

$\langle\delta E\rangle=2 \times 3 / 2 \hbar \omega_{0}$

Ground state energy

Casimir energy

$$
\begin{aligned}
\langle\delta E\rangle & =-\frac{\hbar \alpha(0)^{2}}{c_{0}^{4}} \operatorname{Im} \operatorname{Tr} \int_{0}^{\infty} \frac{d \omega}{2 \pi} \omega^{4} \mathbf{G}^{2}(\omega, \mathbf{r}) \\
& =\frac{\hbar \alpha(0)^{2}}{c_{0}^{4}} \int_{0}^{\infty} \frac{d s}{2 \pi} s^{4} \frac{f\left(i s r / c_{0}\right)^{2}}{(4 \pi r)^{2}}=-\frac{23}{4 \pi} \frac{\hbar c_{0} \alpha(0)^{2}}{(4 \pi)^{2} r^{7}}
\end{aligned}
$$

Casimir Polder interaction energy ${ }^{6}$ 


\subsection{UV catastrophe in sonoluminescence (> 1934)}
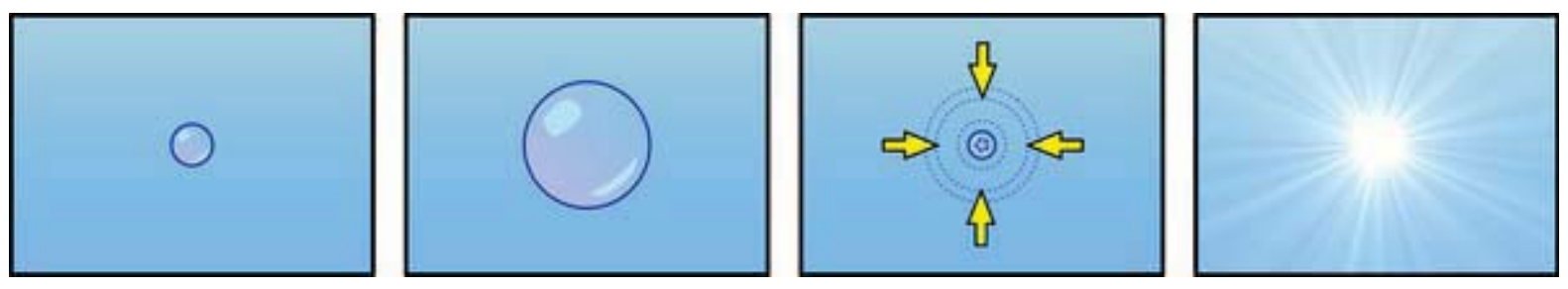

$$
(a=40 \mu m)
$$

\section{Schwinger (1993) 7}

$\Delta E($ bubble $)=\int d^{3} r\left\{\int d^{3} k \frac{1}{2} \hbar \omega_{k}(\right.$ bubble in water $)-\int d^{3} k \frac{1}{2} \hbar \omega_{k}($ water no bubble $\left.)\right\}$

(-)

$$
\approx \frac{\hbar a^{3} \omega_{c}^{4}}{c_{0}^{3}}\left(1-\frac{1}{\sqrt{\varepsilon}}\right) \approx 10 \mathrm{MeV} \longleftarrow \text { cut-off in the UV? }
$$

\section{Dimensional regularisation ${ }^{46}$ ?}

Identity of the van der Waals Force and the Casimir Effect and the Irrelevance of These Phenomena to Sonoluminescence

Iver Brevik*

Division of Applied Mechanics, Norwegian University of Science and Technology, N-7491 Trondheim, Norway

Valery N. Marachevsky"

Department of Theoretical Physics, St. Petersburg University, 198904 St. Petersburg, Russia

Kimball A. Milton ${ }^{3}$

Department of Physics and Astronomy. The University of Oklahoma, Norman, Oklahoma 73019

(Received 12 October 1998)

$$
\begin{aligned}
\langle\delta E\rangle & =\int d^{d>8} \mathbf{r} \frac{-23 \hbar c_{0} \alpha(0)^{2}}{(4 \pi)^{3} r^{7}} \\
& =\frac{+23}{1536 \pi} \frac{\hbar c_{0}(\varepsilon(0)-1)^{2}}{L} \\
& =0.001 \mathrm{eV}
\end{aligned}
$$




\subsection{Casimir energy of dielectric sphere}

$\langle\delta E\rangle=N \times 3 / 2 \hbar \omega_{0}-\frac{1}{2} \sum_{r_{i}, r_{j}<L} \frac{23 \hbar c_{0} \alpha(0)^{2}}{(4 \pi)^{3}\left|\mathbf{r}_{i}-\mathbf{r}_{j}\right|^{7}}$

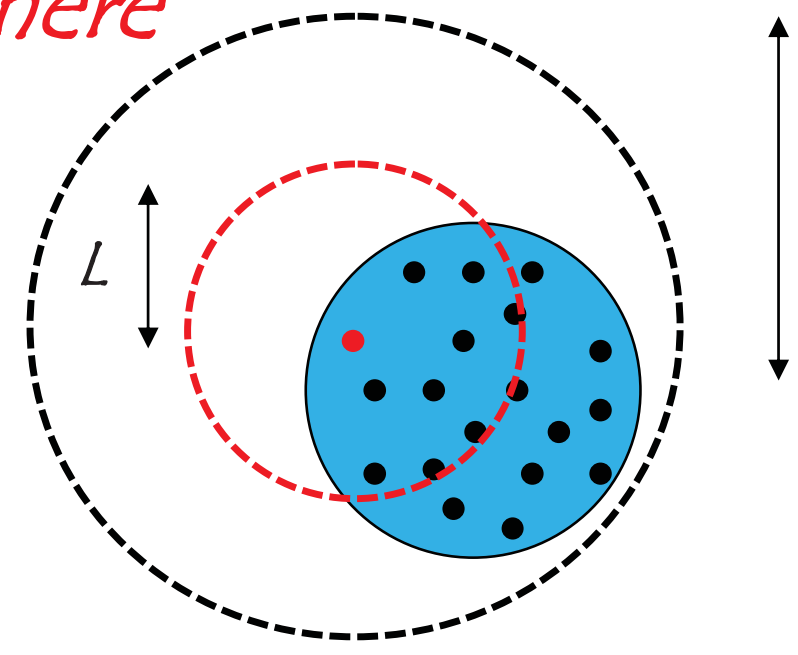

$2 L$

$=N \times 3 / 2 \hbar \omega_{0}-\frac{1}{2} \sum_{r_{i}<L, r_{j}} \frac{23 \hbar c_{0} \alpha(0)^{2}}{(4 \pi)^{3}\left|\mathbf{r}_{i}-\mathbf{r}_{j}\right|^{7}}+\frac{1}{2} \sum_{r_{i}<L, L r_{j}<2 L} \frac{23 \hbar c_{0} \alpha(0)^{2}}{(4 \pi)^{3}\left|\mathbf{r}_{i}-\mathbf{r}_{j}\right|^{7}}+\frac{1}{2} \sum_{r_{i}<L, r_{j}>2 L} \frac{23 \hbar c_{0} \alpha(0)^{2}}{(4 \pi)^{3}\left|\mathbf{r}_{i}-\mathbf{r}_{j}\right|^{7}}$

$=N \times \frac{3}{2} \hbar \omega_{0}+\frac{\hbar c_{0} \alpha(0)^{2}}{(4 \pi)^{2} a^{7}}\left(-0.11 \frac{L^{3}}{a^{3}}+0.4 \frac{L^{2}}{a^{2}}-0.006 \frac{L}{a}\right)+\frac{1}{2} n^{2} \frac{4}{3} \pi L^{3} \int_{r>2 L} d^{3} \mathbf{r} \frac{23 \hbar c_{0} \alpha(0)^{2}}{(4 \pi)^{3} r^{7}}$

Contribution to latent heat 4,44

$$
q[J / k g]=\frac{0.11}{(4 \pi)^{2}} \frac{\hbar c_{0} \alpha(0)^{2}}{a^{7} \times 4 / 3 \pi \rho a^{3}}
$$

$+\frac{23}{1536 \pi} \frac{\hbar c_{0}(\varepsilon(0)-1)^{2}}{L}$

Liquid helium

$$
\alpha(0)=2 A^{3} \quad a=3 A
$$$$
\rho=0.15 \mathrm{~g} / \mathrm{cm}^{3} \Rightarrow q=14 \mathrm{~J} / \mathrm{g}
$$

Regularized Casimir energy stems from missing atoms far outside (Kawka, 2010) 47 
5.7 The magical mystery world of regularization

$$
\begin{gathered}
\int \frac{d^{d} \mathbf{k}}{(2 \pi)^{d}}\left(x^{2}+k^{2}\right)^{-p / 2}=\frac{x^{d-p} \Gamma\left(\frac{p-d}{2}\right)}{(4 \pi)^{d-1} \Gamma\left(\frac{p}{2}\right)}(p>d) \mid \sum_{n=1}^{\infty} \frac{1}{n^{x}}=\zeta(x) \quad(\operatorname{Re} x>1) \\
\Rightarrow P_{\text {casi }}=\frac{\hbar c_{0}}{L} \sum_{n=1}^{\infty} \int \frac{d^{2} \mathbf{k} \mathbf{k}}{(2 \pi)^{d}} \frac{(n \pi / L)^{2}}{\sqrt{(n \pi / L)^{2}+k^{2}}}=\frac{\hbar c_{0}}{4 \pi L} \sum_{n=1}^{\infty} \frac{\Gamma(-1 / 2)}{\Gamma(1 / 2)}\left(\frac{n \pi}{L}\right)^{2} \frac{n \pi}{L} \\
=\frac{\pi^{2}}{4} \frac{\hbar c_{0}}{L^{4}} \frac{\Gamma(-1 / 2)}{\Gamma(1 / 2)} \zeta(-3)=\frac{\hbar c_{0} \pi^{2}}{L^{4}} \frac{-2 \sqrt{\pi}}{4} \frac{1}{\sqrt{\pi}} \frac{1}{120}=-\frac{\pi^{2}}{420} \frac{\hbar c_{0}}{L^{4}} \\
\int_{x<1} d^{d} \mathbf{x} \int_{y<1} d^{d} \mathbf{y} \frac{1}{|\mathbf{x}-\mathbf{y}|^{\gamma}}=\frac{\pi^{d-1 / 2} 2^{d-\gamma} \Gamma\left(\frac{d-\gamma+1}{2}\right)}{(d-\gamma) \Gamma(d / 2) \Gamma(d+1-\gamma / 2)}(d>\gamma / 2) \\
\Rightarrow \int_{x<a} d^{3} \mathbf{x} \int_{y<a} d^{3} \mathbf{y} \frac{-23 \alpha^{2} N^{2}}{4 \pi|\mathbf{x}-\mathbf{y}|^{7}} \underset{\substack{d=3 \\
\gamma=7}}{\longrightarrow}+\frac{23(\varepsilon-1)^{2}}{1536 a}
\end{gathered}
$$

« Regularized 》 Casimir energy of $N$ dipoles distributed in a sphere Brevik etal, 199846 


\subsection{Lifshitz formula Two weakly polarizable bodies 1 and 2} $\langle\delta E\rangle=-\hbar \operatorname{Im} \operatorname{Tr} \int_{0}^{\infty} \frac{d \omega}{2 \pi} \delta t_{1}(\omega) \delta t_{2}(\omega) \mathbf{G}^{2}\left(\omega, \mathbf{r}_{12}\right)$

$$
\begin{aligned}
& =-\hbar \operatorname{Im} \operatorname{Tr} \int_{0}^{\infty} \frac{d \omega}{2 \pi} n_{1} \alpha_{1}(\omega) d \mathbf{r}_{1} \times n_{2} \alpha_{2}(\omega) d \mathbf{r}_{2} \frac{\omega^{4}}{c_{0}^{4}} \mathbf{G}^{2}\left(\omega, \mathbf{r}_{12}\right) \\
& \left\langle E_{\text {casi }}\right\rangle=-\frac{\hbar}{2 \pi c_{0}^{4}} \int d \mathbf{r}_{1} \int d \mathbf{r}_{2} \int_{0}^{\infty} d s s^{4}\left[\varepsilon_{1}(i s)-1\right]\left[\varepsilon_{2}(i s)-1\right] \operatorname{Tr} \mathbf{G}^{2}\left(i s, \mathbf{r}_{12}\right)
\end{aligned}
$$

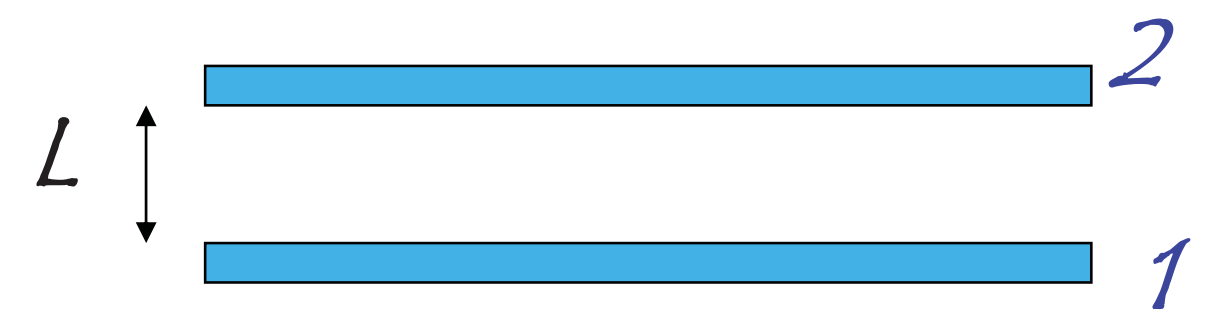

$$
\begin{aligned}
\left\langle E_{\text {casi }}\right\rangle & =-\frac{\hbar}{2 \pi c_{0}^{4}} \int d \mathbf{r}_{1} \int d \mathbf{r}_{2} \int_{0}^{\infty} d s s^{4}\left[\varepsilon_{1}(i s)-1\right]\left[\varepsilon_{2}(i s)-1\right] \operatorname{Tr} \mathbf{G}^{2}\left(i s, \mathbf{r}_{12}\right) \\
& =-\frac{\hbar}{2 \pi c_{0}^{4}} A d^{2} \int d^{2} \mathbf{x} \int_{0}^{\infty} d s s^{4}\left[\varepsilon_{1}(i s)-1 \llbracket\left[\varepsilon_{2}(i s)-1\right] \operatorname{Tr}\left(\frac{1-3 \hat{\mathbf{r}} \hat{\mathbf{r}}}{4 \pi\left(x^{2}+L^{2}\right)^{3 / 2}\left(s / c_{0}\right)^{2}}\right)^{2}\right. \\
& =-\frac{3 \hbar}{2(4 \pi)^{2}} \frac{A d^{2}}{L^{4}} \int_{0}^{\infty} d s\left[\varepsilon_{1}(i s)-1 \llbracket\left[\varepsilon_{2}(i s)-1\right]\right.
\end{aligned}
$$




\subsection{Rigorous Lifshitz formula}

$$
\begin{aligned}
\langle\delta E\rangle & =\frac{\hbar}{2 \pi} \operatorname{Im} \operatorname{Tr} \int_{0}^{i \infty} d(i \zeta) \log \left[1-\mathbf{T}_{1}(i \zeta) \cdot \mathbf{G}_{12}(i \zeta) \cdot \mathbf{T}_{2}(i \zeta) \cdot \mathbf{G}_{21}(i \zeta)\right] \\
& =\frac{\hbar}{2 \pi} \operatorname{Tr} \int_{0}^{\infty} d \zeta \log \left[1-\mathbf{T}_{1}(i \zeta) \cdot \mathbf{G}_{12}(i \zeta) \cdot \mathbf{T}_{2}(i \zeta) \cdot \mathbf{G}_{21}(i \zeta)\right] \\
& =\frac{\hbar}{2 \pi} \int_{0}^{\infty} d \zeta \log \operatorname{det}\left[1-\mathbf{T}_{1}(i \zeta) \cdot \mathbf{G}_{12}(i \zeta) \cdot \mathbf{T}_{2}(i \zeta) \cdot \mathbf{G}_{21}(i \zeta)\right]
\end{aligned}
$$

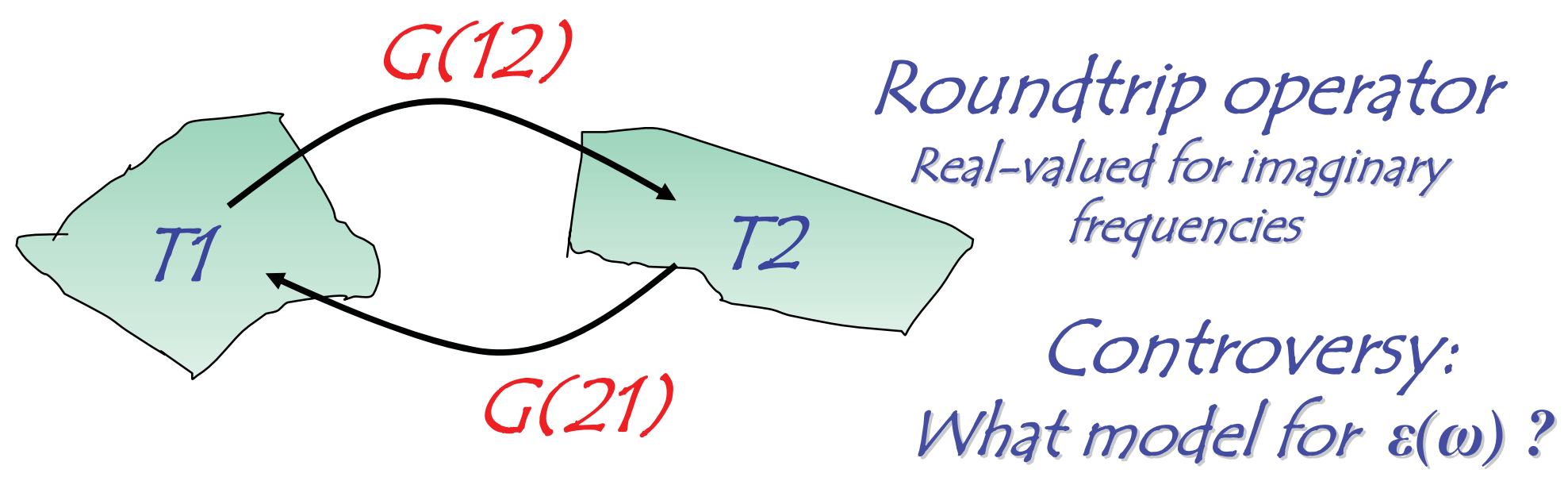


5.10 Thermal Casimir force: Drude or plasma model?

$\varepsilon(\omega)=1-\frac{\omega_{p}^{2}}{\omega^{2}+i \gamma \omega}$

Drude $\varepsilon(\omega)=1-\frac{\omega_{p}^{2}}{\omega^{2}}$

Plasma!

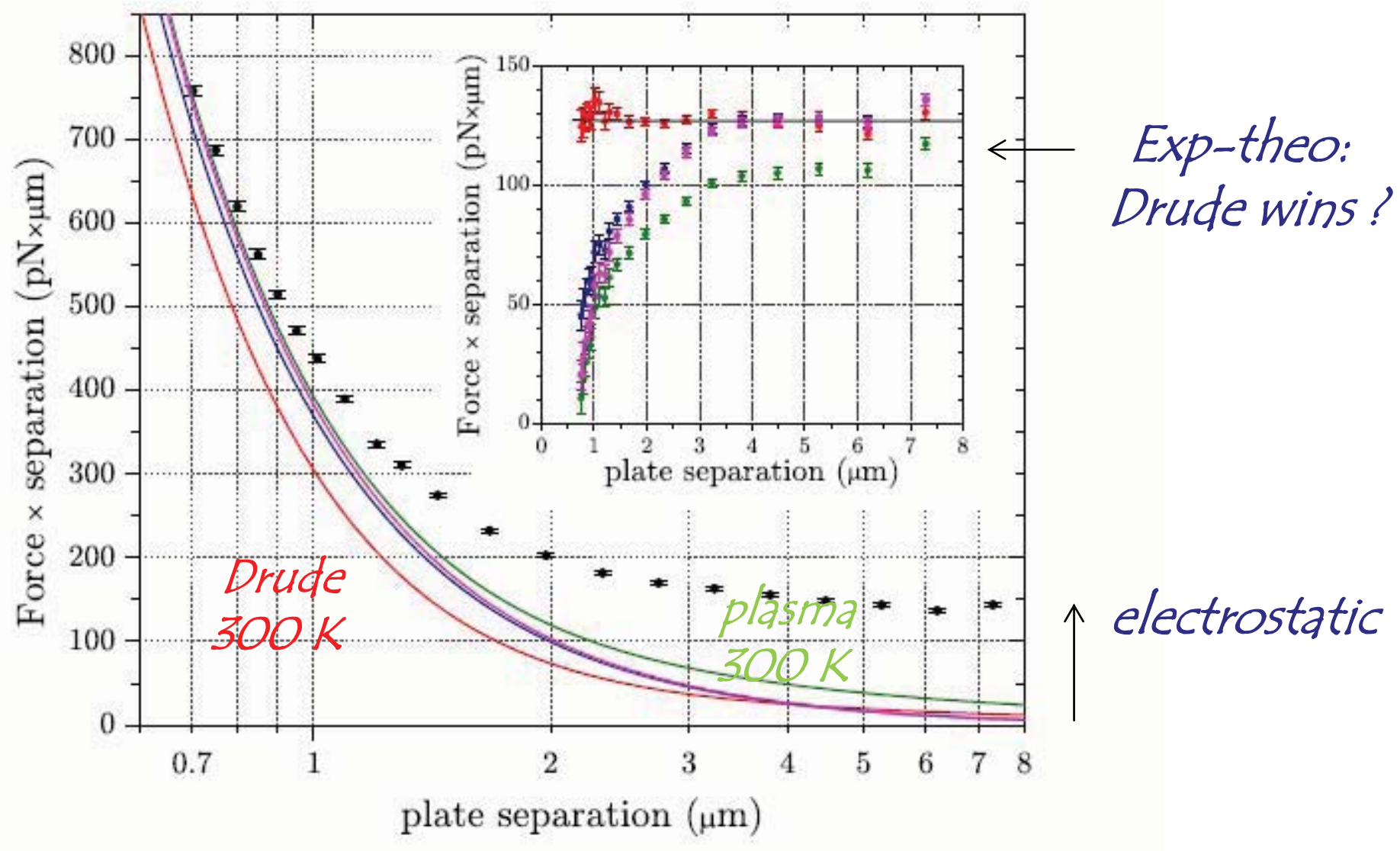

Sushkov, Kim, Dalvit, Lamoreaux, 201139 


\subsection{Proximity Force approximation}

$$
R>>L:\langle\delta E\rangle=-\frac{\pi^{3} \hbar c_{0}}{720} \frac{R}{L^{2}}\left(1-\frac{L}{R}+\ldots\right)
$$

M. Bordag, V. Nikolaev, 200837

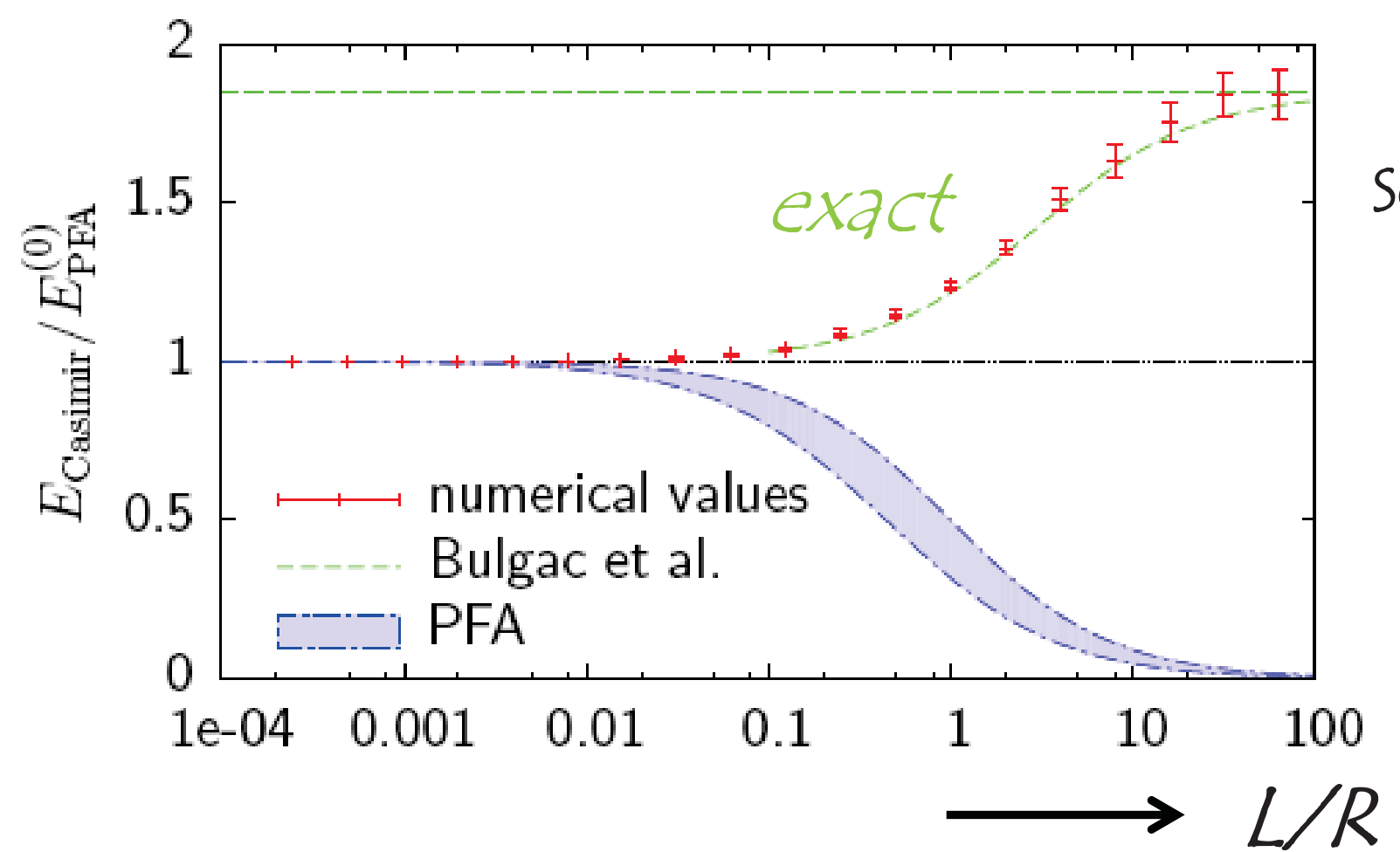

Scalar field with Dirichlet BC's Gies \& Klingmüller, $2006^{48}$ 


\subsection{Casimir force sphere-plane at ambient temperature}

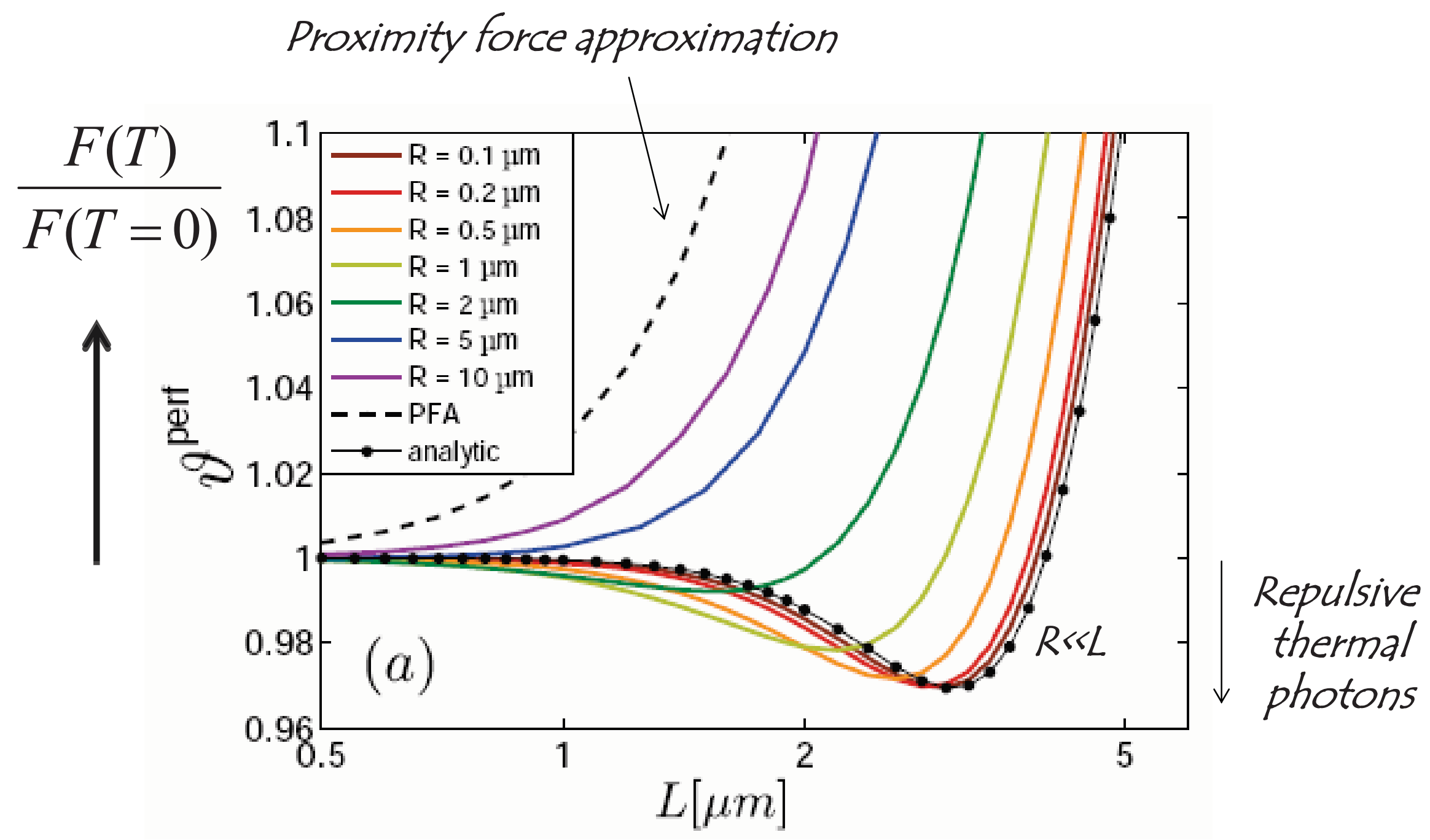

A. Canaguier-Durand, P. A. Maia Neto, A. Lambrecht, S. Reynaud, Phys. Rev. Lett. 104, 040403 (2010). Ref. 36 
6. Quantum vacuum friction: friction or fiction? 


\subsection{Quantum vacuum friction: friction or fiction?}

A little (nonexhaustive) history:

Einstein 1917: friction of moving atom in thermal field (and zero at $\mathrm{T}=0$ ) 3

L Levitov: Van der Waals friction between moving dielectric bodies, Europhys Lett. 198927

J. Pendry 1997 Quantum vacuum shearing J. Phys. Cond Matt. 1997 28: disagrees with Levitov

Dupays, Rizzo etal: quantum friction from vacuum induced magnetic moment by rotating neutron stars, EPL $2008{ }^{49} \rightarrow$ paper seems unnoticed in Casimir community

Philbin etal: macroscopic G-function diagonal: no poor man's friction à la Pendry, 200850

J. Pendry returns: poles of G Doppler shift in complex plane: friction = fact no friction, 201051 (but in fact Ref 50 claims diagonaility throughout the complex plane)

Hoye and Brevik, dissipative quantum friction for $T>0$ between moving oscillators, EPL 2010 52, claims agreement with Barton

Volokitin and Persson (2011) ${ }^{53}$ contest Ref 50 since no excitations. Contested by Ref. $50{ }^{54}$.

G. Barton, Van der Waals friction between atoms and between halfspaces

(claims adreement with Pendry, contests Levitov, contests Hoye \& Brevik) 2010, 201155 
6.3 Quantum vacuum shearing : friction or fiction?
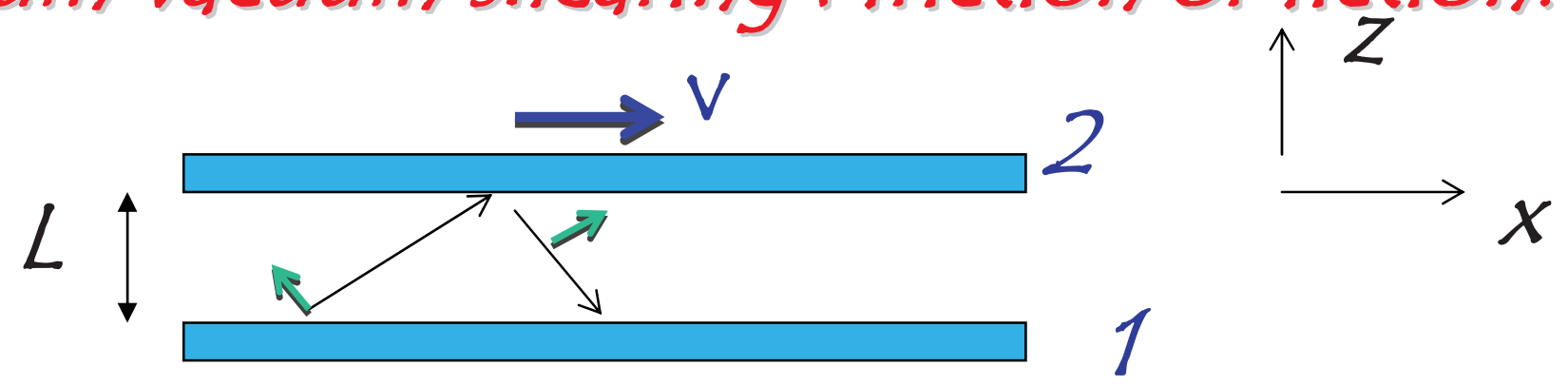

John Pendry, ${ }^{28}$ : friction caused by evanescent low frequency modes

$$
\left\langle T_{x z}\right\rangle \propto\left\langle E_{x}\left(z, \mathbf{k}_{/ /}\right) E_{z}^{*}\left(z, \mathbf{k}_{/ /}\right)\right\rangle \text {for } v \neq 0
$$

$$
\begin{aligned}
F_{x} & =\frac{T_{x z}}{A}=\frac{\hbar}{2} \frac{\int d^{2} \mathbf{k}_{/ /}}{(2 \pi)^{2}} \int_{-\infty}^{+\infty} \frac{d \omega}{2 \pi} \operatorname{sign}(\omega) \exp \left(-k_{/ /} L\right) k_{x} \operatorname{Im} R_{2}\left(\omega+k_{x} v\right) \operatorname{Im} R_{1}(\omega) \\
& =\frac{\hbar}{2(2 \pi)^{2}} \int_{0}^{\infty} d k_{x} \int_{-\infty}^{+\infty} d k_{y} \int_{0}^{k_{x} \mathrm{v}} \frac{d \omega}{2 \pi} \exp \left(-k_{/ /} L\right) k_{x} \operatorname{Im} R_{2}\left(k_{x} \mathrm{v}-\omega\right) \operatorname{Im} R_{1}(\omega)
\end{aligned}
$$

No dissipation $\rightarrow F=O$ 
6.4 Quantum vacuum shearing : friction or fiction?



John Pendry, 28: friction caused by evanescent low frequency modes

$$
\varepsilon(\omega)=1+\frac{\sigma}{-i \omega \varepsilon_{0}} \Rightarrow \frac{F}{A}=\frac{5 \hbar \varepsilon_{0}^{2}}{2^{8} \pi^{2} \sigma^{2}} \frac{\mathrm{v}^{3}}{\mathrm{~L}^{6}}
$$

$\mathrm{v}=1 \mathrm{~m} / \mathrm{s}, \sigma=0.1 / \Omega \mathrm{m}, L=1 \mathrm{~nm} \Rightarrow \mathrm{F} / \mathrm{A}=3 \cdot 10^{-7} \mathrm{~N} / \mathrm{cm}^{2}$

Large!, but...... 
6.5 Quantum vacuum shearing : friction or fiction?



T. Philbin, U. Leonardt, 200950

$$
\begin{gathered}
\left\langle T_{x y}\left(\mathbf{r}_{/ /}, z\right\rangle=\int \frac{d^{2} \mathbf{k}_{/ /}}{(2 \pi)^{2}} \int_{0}^{\infty} \frac{d \omega}{2 \pi}\left\langle\varepsilon_{0} E_{x}\left(z, \mathbf{k}_{/ /}\right) E_{z}^{*}\left(z, \mathbf{k}_{/ /}\right)\right\rangle\right. \\
=-2 \hbar \int \frac{d^{2} \mathbf{k}_{/ /}}{(2 \pi)^{2}} \int_{0}^{\infty} \frac{d \omega}{2 \pi} \frac{\omega^{2}}{c_{0}^{2}} \operatorname{Im} G_{x z}\left(z, z, \omega, \mathbf{k}_{/ /}\right)
\end{gathered}
$$

Green function is diagonal!

\section{No friction?}

Quantizing dissipative media neglects exitations? Real microscopic theory? 


\section{Casimir Momentum}

Work done in collaboration with

Geert Rikken (LNCMI)

James Babington (LPMMC)

Sébastien Kawka (LPMMC)

Support ANR Photonimpuls 


\title{
Quantum Vacuum Contribution to the Momentum of Dielectric Media
}

\author{
A. Feigel* \\ Department of Complex Systems, Weizmann Institute of Science, Rehovot 76100, Israel \\ (Received 3 February 2003; published 16 January 2004)
}

Momentum transfer between matter and electromagnetic field is analyzed. The related equations of motion and conservation laws are derived using relativistic formalism. Their correspondence to various, at first sight self-contradicting, experimental data (the so-called Abraham-Minkowski controversy) is demonstrated. A new, Casimir-like, quantum phenomenon is predicted: contribution of vacuum fluctations to the motion of dielectric liquids in crossed electric and magnetic fields. Velocities of ab ut $50 \mathrm{~nm} / \mathrm{s}$ can be expected due to the contribution of high frequency vacuum modes. The proposed phenomenon conld be used in the future as an investigating tool for zero fluctuations. Other possible applications lie in fields of microfluidics or precise positioning of micro-objects, e.g., cold atoms or molecules.

DOI: 10.1103/PhysRevLett.92.020404

PACS numbers: 03.50.De, $42.50 . \mathrm{Nn}, 42.50 . \mathrm{Vk}$
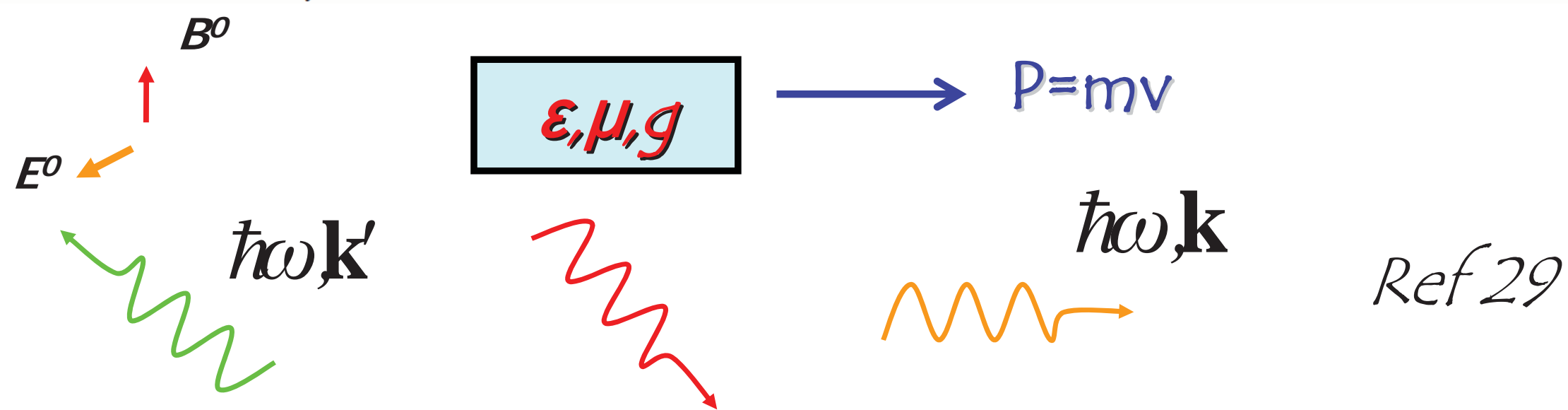
7.3 Bi-anisotropic Media

Fresnel dispersion law

$$
\begin{aligned}
& \mathbf{D}(\omega)=\varepsilon(\omega) \mathbf{E}(\omega)+\mathbf{g}(\omega) \cdot \mathbf{B}(\omega) \\
& \mathbf{H}(\omega)=\mathbf{g}^{T}(\omega) \cdot \mathbf{E}(\omega)+\mu(\omega)^{-1} \mathbf{B}(\omega)
\end{aligned}
$$

$\operatorname{det}\left(\varepsilon \frac{\omega^{2}}{c_{0}^{2}}-k^{2}+\mathbf{k k}-\frac{\omega}{c_{0}} \mathbf{g} \cdot(\boldsymbol{\varepsilon} \cdot \mathbf{k})+\frac{\omega}{c_{0}}(\boldsymbol{\varepsilon} \cdot \mathbf{k}) \cdot \mathbf{g} *\right)=0$

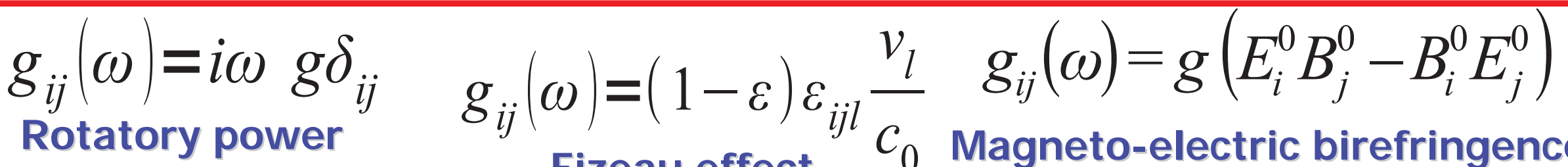

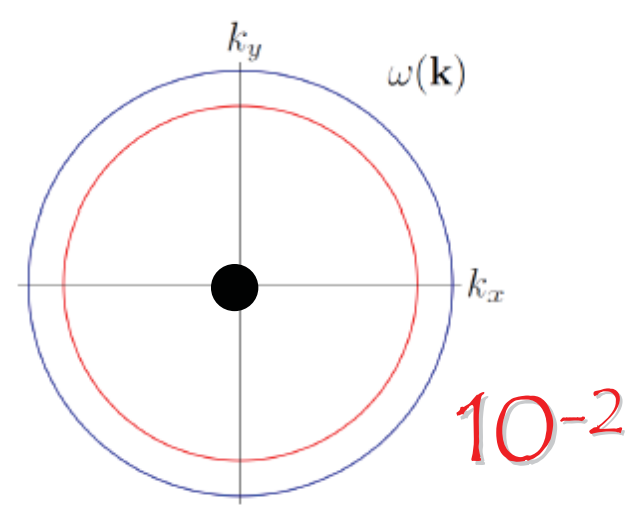

Fizeau effect $c_{0}$ Magneto-electric birefringence

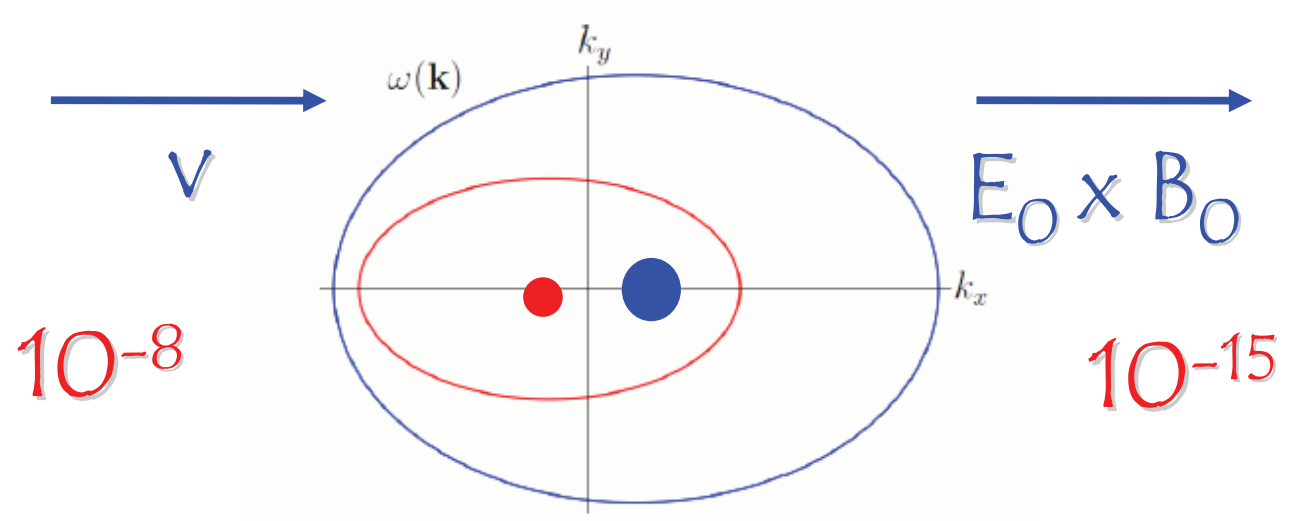




\section{4 phenomenological continuum theory}

$$
\begin{aligned}
& \partial_{t}\left(\rho \mathbf{v}+\varepsilon_{0} \mathbf{E} \times \mathbf{B}\right)=-\nabla \cdot \mathbf{T}^{0} \\
& T_{i j}^{0}=\frac{1}{8 \pi}\left(\varepsilon_{0} E^{2}+\frac{1}{\mu_{0}} B^{2}\right) \delta_{i j}-\frac{1}{4 \pi}\left(\varepsilon_{0} E_{i} E_{j}+\frac{1}{\mu_{0}} B_{i} B_{j}\right)
\end{aligned}
$$

$\left\langle 0\left|\frac{\mathbf{E} \times \mathbf{B}}{4 \pi c_{0}}\right| 0\right\rangle \propto\left\{\begin{array}{l}\frac{1}{c_{0}} \int d^{3} \mathbf{k} \frac{1}{2} \hbar \omega_{k} \times g(\omega) \mathbf{E}_{0} \times \mathbf{B}_{0}=\frac{2}{3} \frac{\hbar \omega_{c}^{4}}{\pi^{3} c_{0}^{4}} g \mathbf{E}_{0} \times \mathbf{B}_{0} \\ \frac{1}{c_{0}} \int d^{3} \mathbf{k} \frac{1}{2} \hbar \omega_{k} \times[\varepsilon(\omega)-1] \frac{\mathbf{v}}{c_{0}}=\rho_{\text {casi }} \mathbf{v}\end{array}\right.$

Photonic momentum in dielectric media?

$\rightarrow$ classical "Abraham » contribution already controversial UV catastrophe of vacuum energy? Lorentz invariance of quantum vacuum? Inertia of quantum vacuum?

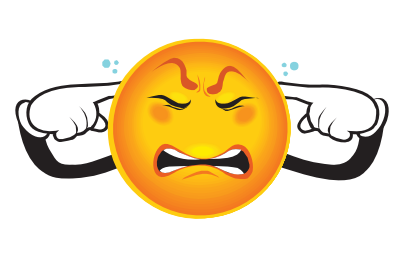




\subsection{Casimir momentum not excluded by Lorentz invariance}

$$
L(\mathbf{E}, \mathbf{B})=\frac{1}{2}\left(\mathbf{E}^{2}-\mathbf{B}^{2}\right)+2 v\left(\mathbf{E}^{2}-\mathbf{B}^{2}\right)^{2}+\frac{v}{2}(\mathbf{E} \cdot \mathbf{B})^{2}
$$

$$
\begin{aligned}
& \mathbf{E}=\mathbf{E}_{0}+\mathbf{E}(\omega) \\
& \mathbf{B}=\mathbf{B}_{0}+\mathbf{B}(\omega)
\end{aligned} \quad \begin{gathered}
\text { Bit-anisotropic } \\
\text { Lorentz-ipvariant vacuum }
\end{gathered}
$$

FluctuationDissipation

$\left\langle 0\left|E_{i}(\mathbf{r}, \omega) E_{j}^{*}\left(\mathbf{r}^{\prime}, \omega^{\prime}\right)\right| 0\right\rangle=-2 \hbar \omega^{2} \operatorname{ImG}_{i j}\left(\mathbf{r}, \mathbf{r}^{\prime}, \omega\right) \times 2 \pi \delta\left(\omega-\omega^{\prime}\right)$

$\left\langle 0\left|\frac{c_{0}}{4 \pi} \mathbf{E}^{*} \times \mathbf{H}\right| 0\right\rangle=0$

Zero energy flow

Rikken \& Rizzo (2003) 56 Van Tiggelen, Rikken, 200957

$$
\left\langle 0\left|\frac{\mathbf{E}^{*} \times \mathbf{B}}{4 \pi c_{0}}\right| 0\right\rangle=-\frac{4}{3} v K \mathbf{E}_{0} \times \mathbf{B}_{0}
$$

infinite momentum density

$$
K=\frac{1}{(2 \pi)^{3}} 1 / 2 \hbar \int_{0}^{\infty} d \omega \int_{4 \pi} d \Omega \rho_{0}(\omega, \Omega)
$$
Infinite Lorentz scalar 


\section{6 ........but does it satisfy general relativity?}

$$
R_{\mu \nu}-R g_{\mu \nu}+\Lambda g_{\mu \nu}=-\frac{8 \pi G}{c_{0}^{4}} T_{\mu \nu} \Rightarrow T_{\mu \nu}=T_{\nu \mu}
$$

\begin{tabular}{|c|c|c|}
\hline$T_{\nu \mu}=$ & $\begin{array}{l}(\mathbf{E} \times \mathbf{B})_{\text {casi }} E_{\text {casi }} \\
\quad=-4 / 3 v E_{\text {casi }}\left(\mathbf{E}_{0} \times \mathbf{B}_{0}\right) \\
\quad \neq 0\end{array}$ & $\begin{array}{c}(\mathbf{E} \times \mathbf{H})_{c a s i}=0 \\
{\left[T_{i j}\right]_{c a s i}}\end{array}$ \\
\hline
\end{tabular}


7.7 The Abraham Force

Macroscopic

$\longrightarrow \partial_{t} \mathbf{G}_{M}+\nabla \cdot \mathbf{T}=\mathbf{f}$

$$
\begin{aligned}
& \mathbf{G}_{M}=\mathbf{D} \times \mathbf{B} \\
& \mathbf{f}=-E^{2} \nabla \varepsilon-H^{2} \nabla \mu \\
& \text { Minkowski }
\end{aligned}
$$




\subsection{The Abraham Force (see Brevik ${ }^{58}$ )}

$\mathbf{G}_{A}=\varepsilon_{0} \mu_{0} \mathbf{E} \times \mathbf{H}$

$=\frac{1}{c_{0}} \mathbf{S}$

$\partial_{t} \mathbf{G}_{A}+\nabla \cdot \mathbf{T}=\mathbf{f}-\varepsilon_{0}\left(\varepsilon_{r}-1 / \mu_{r}\right) \partial_{t}(\mathbf{E} \times \mathbf{B})$ Abraham ${ }^{58}$

$\mathbf{G}_{N}=\varepsilon_{0} \mathbf{E} \times \mathbf{B}$

$=\mathbf{G}_{0}$

$\partial_{t} \mathbf{G}_{N}+\nabla \cdot \mathbf{T}=\mathbf{f}-\varepsilon_{0}\left(\varepsilon_{r}-1\right) \partial_{t}(\mathbf{E} \times \mathbf{B}) \quad$ Nelson ${ }^{59}$

$\ldots=-\mathbf{f}-\frac{2}{5}\left[\partial_{t} \mathbf{E}_{0}(t)\right] \times \mathbf{B}_{0}(t)-\mathbf{E}_{0}(t) \times\left[\partial_{t} \mathbf{B}_{0}(t)\right] \quad$ Peierls 60

$\exp \quad \mathbf{F}=(\varepsilon-1) V \partial_{t} \mathbf{E}_{0}(t) \times \mathbf{B}_{0}(t) \quad$ Walker \& Walker,

theo

Abraham momentum = kinetic momentum, Minkowski momentum = conjugate momentum

Nelson momentum $=$ pseudo momenturp
Barnett

(2010) 62

Nelson

(1991) 59 


\subsection{The Abraham Force (Nelson version)}

$\underset{\substack{\text { Macrossopic } \\ \text { Maxwell }}}{-} \quad \partial_{t}(\mathbf{D} \times \mathbf{B})-\partial_{t}(\mathbf{P} \times \mathbf{B})+\nabla \cdot \mathbf{T}=\mathbf{f}-\partial_{t}(\mathbf{P} \times \mathbf{B})$

$$
\mathbf{f}=-E^{2} \nabla \varepsilon-H^{2} \nabla \mu
$$

Maxwell-Lorentz force on induced polarization and current

$$
\partial_{t} \rho \mathbf{v}+\nabla \cdot \mathbf{U}=\mathbf{f}+\partial_{t}(\mathbf{P} \times \mathbf{B})
$$

Microscopic Maxwell

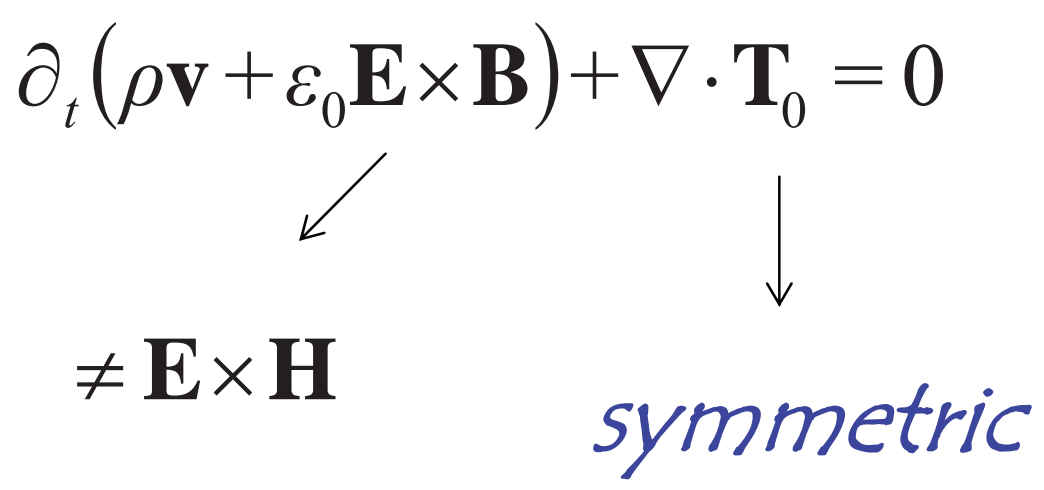




\subsection{Classical Abraham momentum in crossed EM fields}

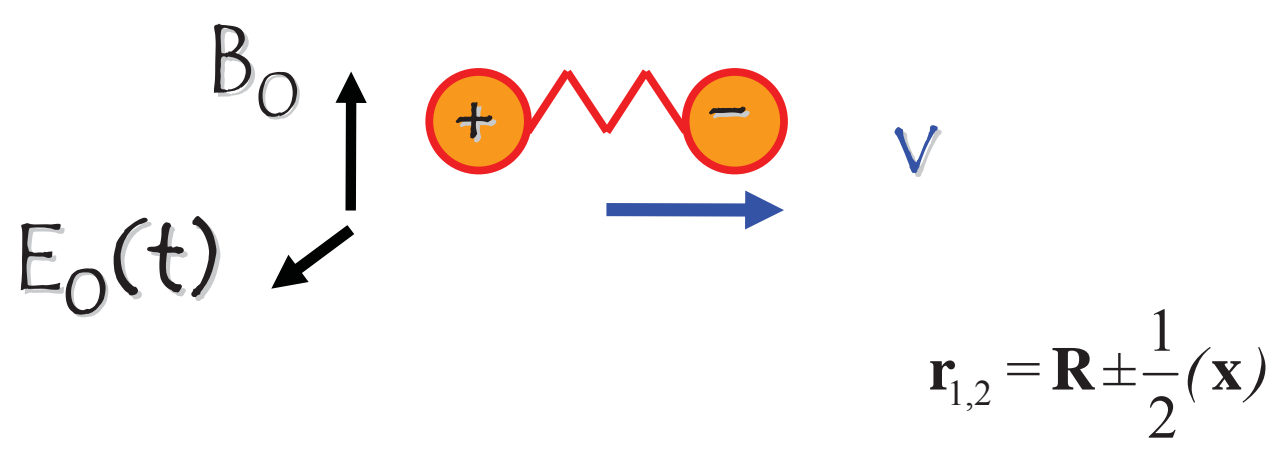

$m \ddot{\mathbf{m}_{1}}=+q \mathbf{E}(t)+q \dot{\mathbf{r}_{1}} \times \mathbf{B}+\mathbf{f}\left(r_{12}\right) \quad 2 \mathrm{~m} \dot{\mathbf{R}}+q \mathbf{x} \times \mathbf{B}=$ constan $=0$

$m \ddot{\mathbf{r}_{2}}=-q \mathbf{E}(t)-q \dot{\mathbf{r}}_{2} \times \mathbf{B}-\mathbf{f}\left(r_{12}\right) \quad m \ddot{\mathbf{x}}=2 \mathrm{q} \mathbf{E}(t)+2 \mathrm{q} \dot{\mathbf{R}} \times \mathbf{B}-m \omega_{0}^{2} \mathbf{x} \approx 0$

$$
2 \mathrm{~m} \dot{\mathbf{R}}=\frac{q^{2} / m}{\omega_{0}^{2}} \mathbf{E}_{0}(t) \times \mathbf{B}_{0}
$$

No controversy exists in microscopic description Consistent with Abrahams and Nelson version

$$
\frac{q^{2} / m}{\varepsilon_{0} \omega_{0}^{2}}=\alpha(0)=\frac{\varepsilon-1}{n}
$$




\subsection{The UV catastrophe is real in macroscopic description} Free electron (electric dipole) 2

$$
\varepsilon(\omega)-1=-\frac{\omega_{P}^{2}}{\omega^{2}} \quad \rho_{\text {casi }}=\frac{\hbar}{c_{0}^{3}} \int_{0}^{\infty} d \omega \omega^{3} \frac{\omega_{p}^{2}}{\omega^{2}}=\infty
$$

$g_{M E}(\boldsymbol{\omega})=-\frac{e^{4} m_{\Delta}^{2}}{\omega_{0}^{2} m^{3} M^{2}}\left[-\frac{\omega^{2}+\omega_{0}^{2}}{\left(\omega_{0}^{2}-\omega^{2}\right)^{2}}\left(\mathbf{E}_{i}^{0} \mathbf{B}_{j}^{0}-\left(\mathbf{E}^{0} \cdot \mathbf{B}^{0}\right) \delta_{i j}\right)\right.$

$$
\left.+\frac{1}{\omega_{0}^{2}-\omega^{2}}\left(\mathbf{E}_{i}^{0} \mathbf{B}_{j}^{0}-\frac{1}{4} \mathbf{E}_{j}^{0} \mathbf{B}_{i}^{0}-\frac{1}{4}\left(\mathbf{E}^{0} \cdot \mathbf{B}^{0}\right) \delta_{i j}\right)\right]
$$

$$
P_{\text {casi }}=\frac{\hbar}{c_{0}^{3}} \int d r \int_{0}^{\infty} d \omega \omega^{3} g(\omega) E_{0} \times B_{0}=\infty
$$

Electric quadrupole

$$
\mathbf{P}_{\text {casi }}=\frac{\hbar c_{0} g(\varepsilon(0)-1)}{a} \mathbf{E}_{0} \times \underset{\text { Dimensio }}{\mathbf{B}_{0} ?}
$$

Rizzo etal, 2003, 2009 63, Babington \& BAvT, 2011, ${ }^{64}$ 


\subsection{Observation of the Abraham Force}

Ex: Helium

$$
\begin{aligned}
& E O=450 \mathrm{~V} / \mathrm{mm} ; B O=1 T \\
& \alpha(0)=0.2210^{-40} \mathrm{Cm}^{2} / \mathrm{V} \quad\left(16.6 \mathrm{a}_{0}^{3}\right) \\
& \rho=0.17 \mathrm{~kg} / \mathrm{m}^{3}(\operatorname{room~} T) \\
& g=0.01710^{-22} \mathrm{~m} / \mathrm{VT}
\end{aligned}
$$

(S/ units)

$v_{\mathrm{abr}}=\frac{\varepsilon_{0} \alpha(0) E B}{2 \mathrm{~m}_{p}} \approx 0.3 \mathrm{~nm} / \mathrm{sec}$

$v_{\text {Feigel }}=\frac{\pi}{4} \frac{h}{\rho \lambda_{c}^{4}} g E B \approx 0.02 \mathrm{~nm} / \mathrm{sec}$

$\mathrm{v}_{Q E D} \propto \mathrm{v}_{\mathrm{abr}} \times(\mathrm{Z} ! \alpha)^{2} \approx 0.001 \mathrm{~nm} / \mathrm{sec}$
Classical Abraham

Force

$$
\begin{aligned}
& F_{\mathrm{abr}} \approx 7 \cdot 10^{-32} \mathrm{~N} \\
& N_{a t} F_{\mathrm{abr}} \propto 10^{-13} \mathrm{~N}
\end{aligned}
$$

Semi-classical QED with cut-off $0.1 \mathrm{~nm}$ (Feigel ${ }^{29}$ )

Rigorous QED (Kawka, 2010 66) 


$$
\frac{d p}{d t}=\alpha(0) \frac{d E}{d t} \times B
$$

Acoustic $\quad P(\omega)=P_{0}+\alpha(0) \times E \times B \times \omega \times \cos \omega t \times n \times L$ pressure
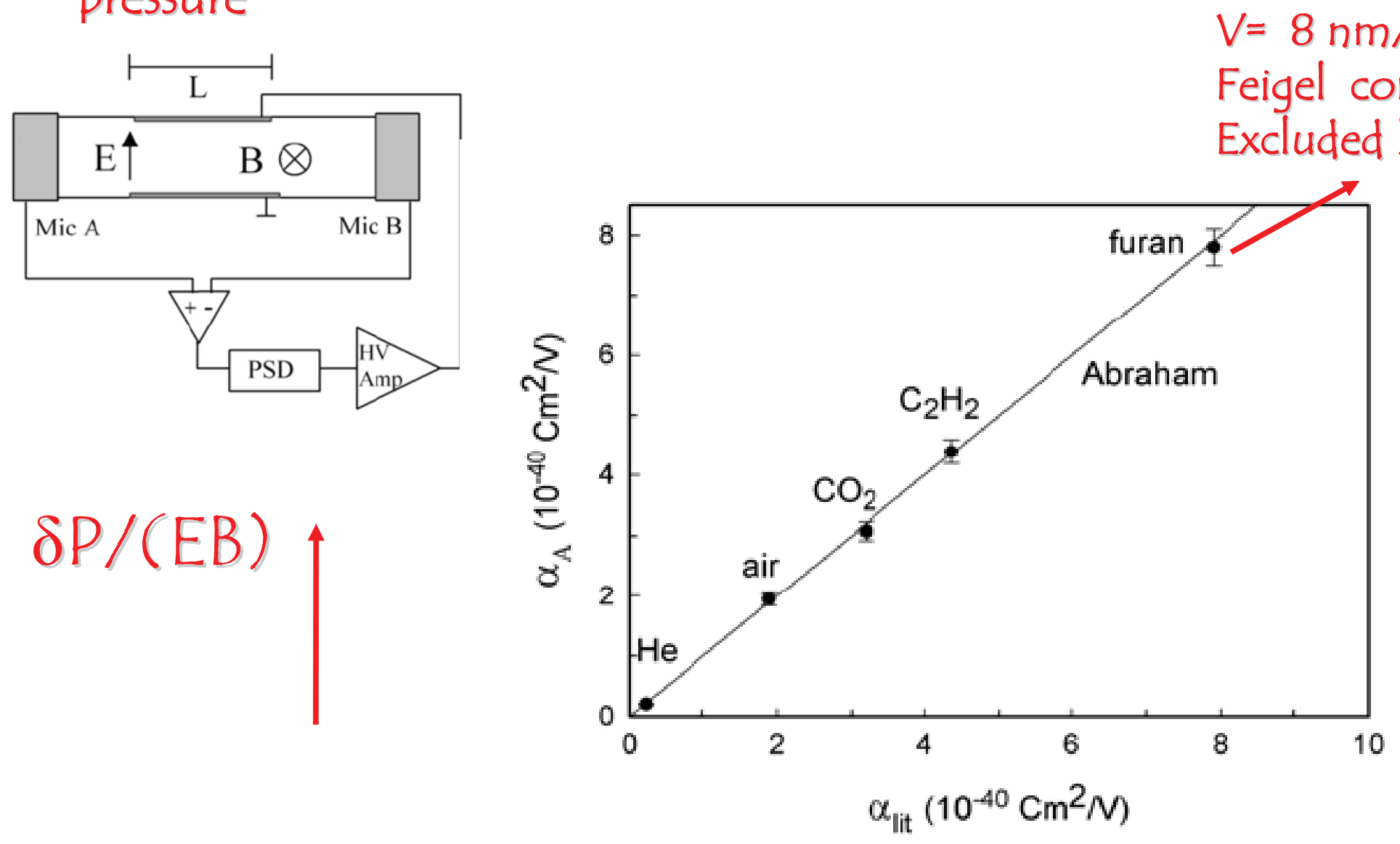

$\mathrm{E}=450 \mathrm{~V} / \mathrm{mm}$; $\mathrm{B}=1 \mathrm{~T}$;

$f=7.6 \mathrm{kHz}$

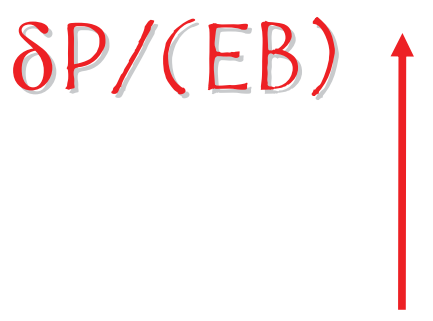

$$
\alpha_{\text {lit }}\left(10^{-40} \mathrm{Cm}^{2} / \mathrm{N}\right)
$$

Rikken / Van Tiggelen, 201167 


\section{Casimir momentum: 1/6 QED of atom in crossed fields}

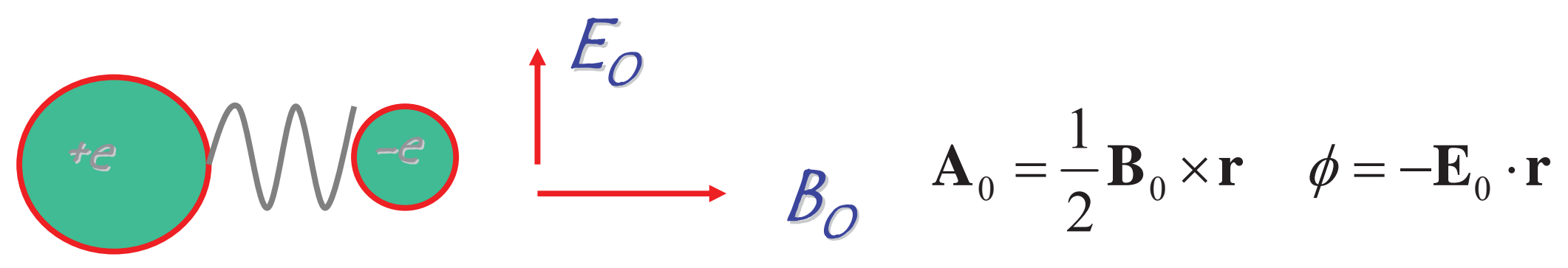

\section{Coulomb Gauge}

$$
\begin{gathered}
H=\frac{1}{2 m_{1}}\left(\mathbf{p}_{1}-e \mathbf{A}_{0}\left(\mathbf{r}_{1}\right)-e \mathbf{A}\left(\mathbf{r}_{1}\right)\right)^{2}+\frac{1}{2 m_{2}}\left(\mathbf{p}_{2}+e \mathbf{A}_{0}\left(\mathbf{r}_{2}\right)+e \mathbf{A}\left(\mathbf{r}_{2}\right)\right)^{2} \\
+e \mathbf{E}_{0} \cdot \mathbf{r}_{21}+V\left(r_{12}\right)
\end{gathered}
$$

$$
+\sum_{i} \hbar \omega_{i}\left(a_{i}^{*} a_{i}+1 / 2\right)
$$


Casimir momentum: $2 / 6$

QED of atom in crossed fields

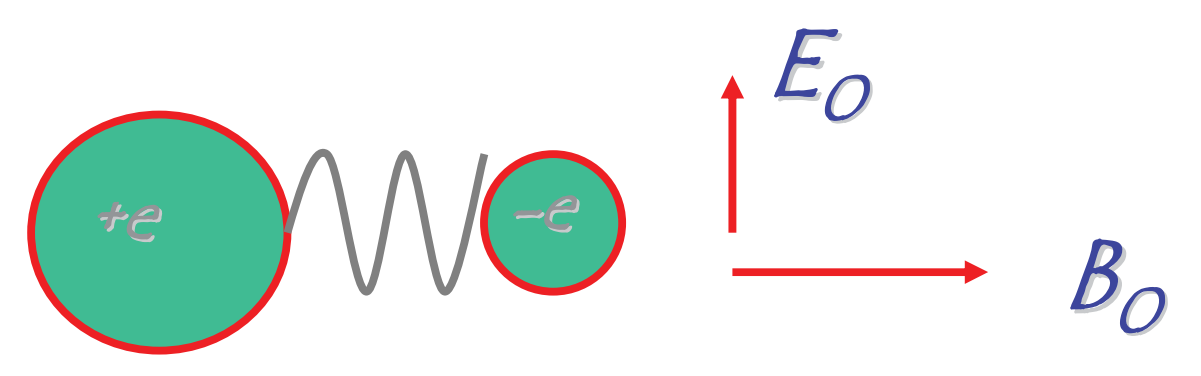

Conjugate momenta

* kinetic momentum

$$
\begin{aligned}
& \mathbf{p}_{1}=m_{1} \mathbf{v}_{1}+e \mathbf{A}_{0}\left(\mathbf{r}_{1}\right) \\
& \mathbf{p}_{2}=m_{1} \mathbf{v}_{2}-e \mathbf{A}_{0}\left(\mathbf{r}_{2}\right)
\end{aligned}
$$

Pseudo momentum

$$
\hat{\mathbf{K}}=\mathbf{p}_{1}+\mathbf{p}_{2}+\frac{1}{2} e \mathbf{B}_{0} \times \mathbf{r}_{21}=\mathbf{P}_{k i n}+e \mathbf{B}_{0} \times \mathbf{r}
$$

Pseudo momentum is conserved

$[\mathbf{K}, H]=0$ 
Ground state changes due to coupling with quantum vacuum

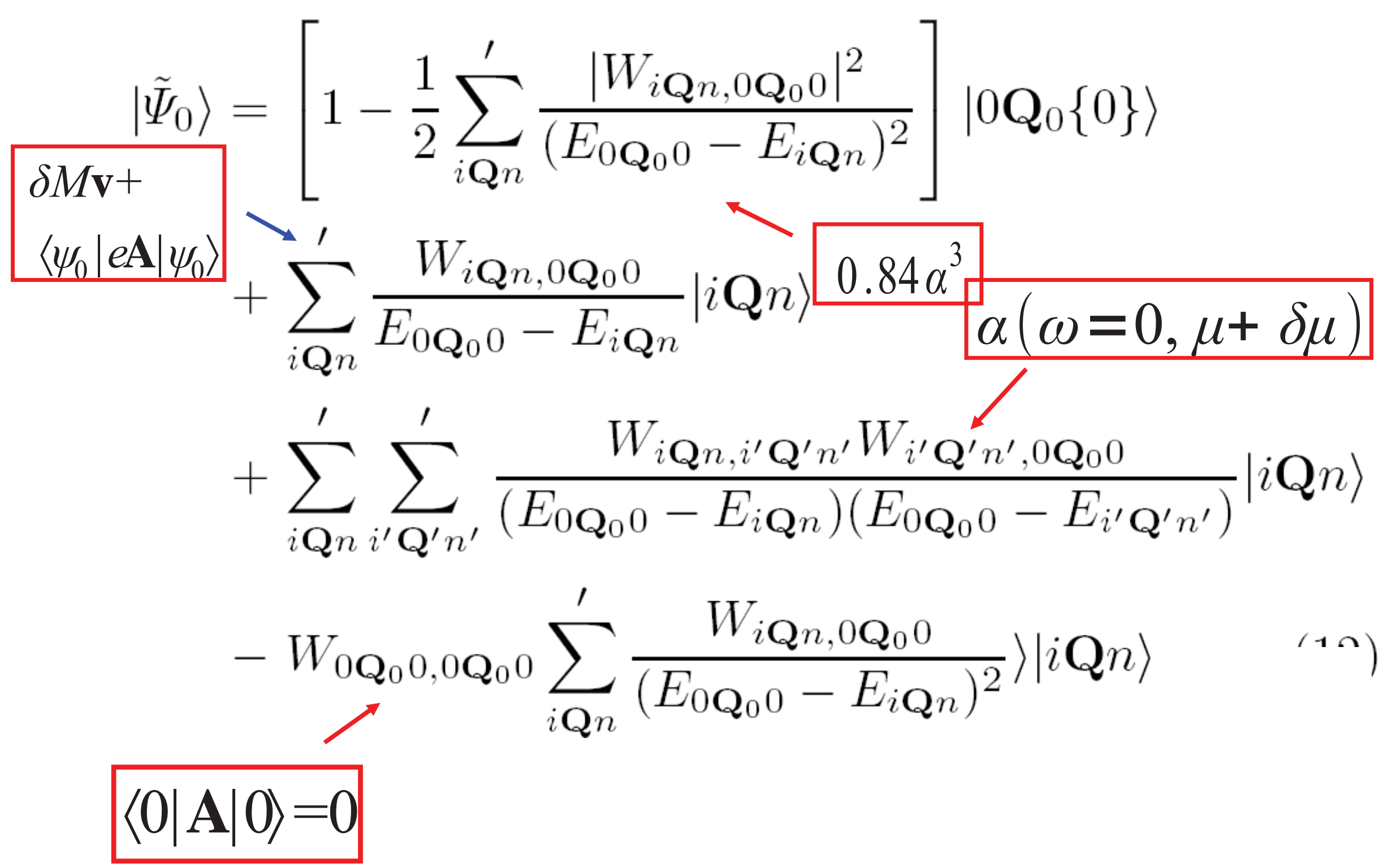


Casimir momentum: 3/6

QED of hydrogen atom in crossed fields

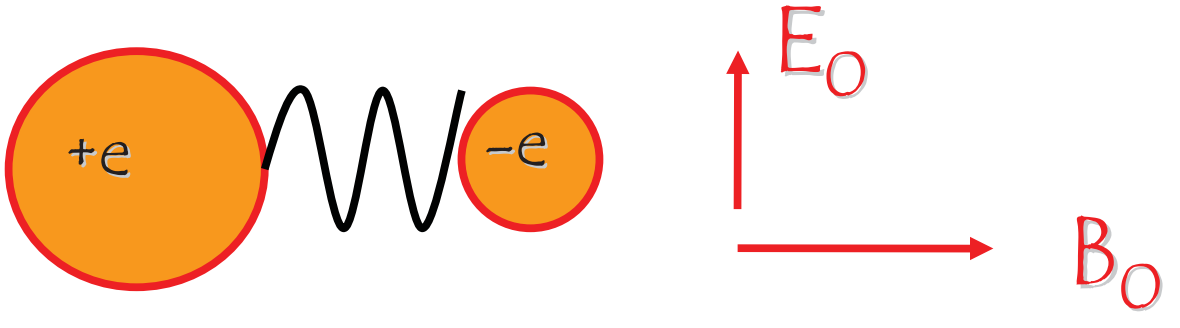

$\hat{\mathbf{K}}=m_{1} \mathbf{v}_{1}+m_{2} \mathbf{v}_{2}+e \mathbf{A}\left(\mathbf{r}_{1}\right)-e \mathbf{A}\left(\mathbf{r}_{2}\right)+e \mathbf{B}_{0} \times \mathbf{r}_{21}+\sum_{i} \hbar \mathbf{k}_{i}\left(a_{i}{ }^{*} a_{i}+12\right)$

No multipole approximation in $\quad \mathbf{A}(\mathbf{r}) \propto \sum_{g k} \mathbf{g}_{k} \exp (i \mathbf{k r} \mathbf{r}) a_{\mathbf{g k}}+c . c$

$\left\langle\Psi_{0}|\mathbf{K}| \Psi_{0}\right\rangle=M \mathbf{v}+\delta M \mathbf{v}+\frac{8}{3} \frac{E_{0}}{c_{0}^{2}} \mathbf{v}$

$+\varepsilon_{0} \alpha(0) \mathbf{B}_{0} \times \mathbf{E}_{0}+\varepsilon_{0} \delta \mu \partial_{\mu} \alpha(0) \mathbf{B}_{0} \times \mathbf{E}_{0}+\mathbf{K}_{1}+\mathbf{K}_{2}$

$\delta M=\delta\left(m_{1}+m_{2}\right) \quad \delta \mu=\delta\left(\frac{m_{1} m_{2}}{m_{1}+m_{2}}\right) \quad \delta m_{i}=\frac{4}{3 \pi} \alpha \hbar \int_{0}^{\infty} d k \frac{\hbar k}{\hbar^{2} k^{2} / 2 \mathrm{~m}_{i}+\hbar k c}$ 


\section{Casimir momentum: 416}

QED of hydrogen atom in crossed fields

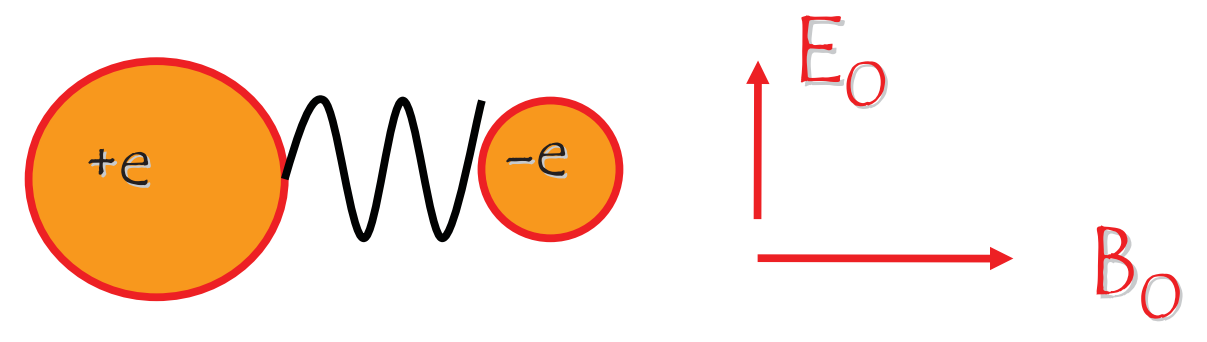

$$
\begin{aligned}
& \hat{\mathbf{K}}=m_{1} \mathbf{v}_{1}+m_{2} \mathbf{v}_{2}+e \mathbf{A}\left(\mathbf{r}_{1}\right)-e \mathbf{A}\left(\mathbf{r}_{2}\right)+e \mathbf{B}_{0} \times \mathbf{r}_{21}+\sum_{i} \hbar \mathbf{k}_{i}\left(a_{i}{ }^{*} a_{i}+\frac{1}{2}\right) \\
& -\frac{m_{1}^{2} \mathbf{v}_{1}^{2}}{2 \mathrm{c}_{0}^{2}} \mathbf{v}_{1}+\text { idem } 2 \\
& \text { Atomic binding energy } \\
& \text { dominated by Casimir energy } \\
& \left\langle\Psi_{0}|\mathbf{K}| \Psi_{0}\right\rangle=M \mathbf{v}+\delta M \mathbf{v}+\frac{8}{3} \frac{E_{0}}{c_{0}^{2}} \mathbf{v}-\frac{5}{3} \frac{E_{0}}{c_{0}^{2}} \mathbf{v} \\
& +\varepsilon_{0} \alpha(0) \mathbf{B}_{0} \times \mathbf{E}_{0}+\delta(\mu) \varepsilon_{0} \partial_{\mu} \alpha(0) \mathbf{B}_{0} \times \mathbf{E}_{0} \\
& +\mathbf{K}_{1}+\mathbf{K}_{2}+\mathbf{K}_{R} \\
& \delta M=\delta\left(m_{1}+m_{2}\right) \quad \delta \mu=\delta\left(\frac{m_{1} m_{2}}{m_{1}+m_{2}}\right) \quad \delta m_{i}=\frac{4}{3 \pi} \alpha \hbar \int_{0}^{\infty} d k \frac{\hbar k}{\hbar^{2} k^{2} / 2 \mathrm{~m}_{i}+\hbar k c}
\end{aligned}
$$




\section{Casimir momentum: 5/6}

QED of hydrogen in crossed fields

$$
\begin{aligned}
& \text { Quantum vacuum contribution: } \\
& \mathbf{K}_{1}=-\mathbf{B}_{0} \times \mathbf{E}_{0} \frac{1}{3} \frac{e^{2} \hbar^{2}}{a_{0} c_{0}^{2} \mu^{2}} \sum_{n}\left\langle 0\left|\frac{e^{2}}{4 \pi \varepsilon_{0} r} \hat{\mathbf{r}}\right| n\right\rangle \cdot \frac{1}{\left(E_{n}-E_{0}\right)^{2}} \cdot\langle n|\mathbf{r}| 0\rangle \\
& =-\varepsilon_{0} \alpha(0) \mathbf{B}_{0} \times \mathbf{E}_{0} \alpha^{2}(0.208+0.0045)=-0.21 \alpha^{2} \mathbf{K}_{A} \\
& \mathbf{K}_{2}=+\varepsilon_{0} \alpha(0) \mathbf{B}_{0} \times \mathbf{E}_{0} \alpha^{2} \frac{1}{27} \frac{e^{2}}{4 \pi \varepsilon_{0} a_{0}^{2}} \sum_{n}\langle 0|\hat{\mathbf{r}}| n\rangle \cdot \frac{1}{E_{n}-E_{0}} \cdot\langle n|\mathbf{r}| 0\rangle \\
& =+\varepsilon_{0} \alpha(0) \mathbf{B}_{0} \times \mathbf{E}_{0} \alpha^{2}(0.079+0.018)=+0.1 \alpha^{2} \mathbf{K}_{A} \\
& \text { Continuous spectrum } \\
& \text { assuming } \\
& \text { plane waves for electrons }
\end{aligned}
$$

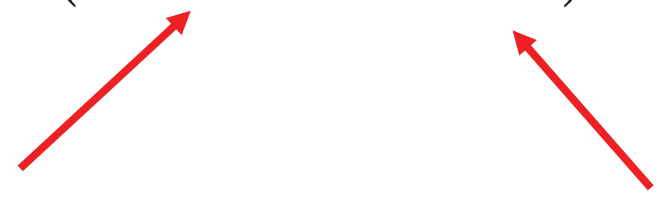


Casimir momentum: $6 / 6$

QED of hydrogen in crossed fields

$\overbrace{\text { Relativistic contribution: }}^{\longrightarrow} \mathrm{B}_{\mathrm{O}}$

$$
\begin{aligned}
& \mathbf{K}_{R}=-\frac{e^{2}}{2 \mathrm{~m}_{e} M c_{0}^{2}}\left\langle 0_{E}\left|p^{2}\left(\mathbf{B}_{0} \times \mathbf{x}\right)\right| 0_{E}\right\rangle \\
& \propto \alpha^{2} \frac{m_{e}}{M} \mathbf{K}_{A}
\end{aligned}
$$


Casimir momentum: $6 / 6$ QED of hydrogen in crossed fields

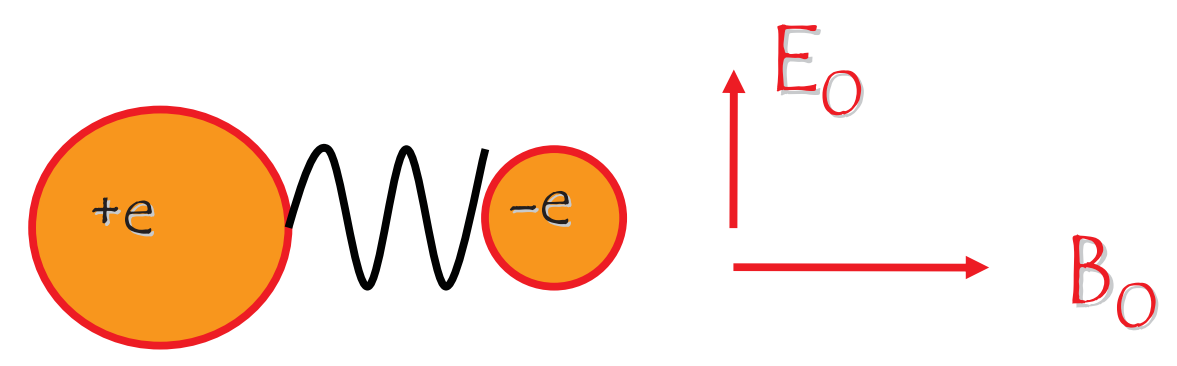

$$
\begin{gathered}
\langle\mathbf{K}\rangle=\left(m_{e}+m_{p}\right) \mathbf{v}+\frac{E_{0}}{\underline{c_{0}^{2}} \mathbf{v}}+\mathbf{K}_{A}-0.1 \alpha^{2} \mathbf{K}_{A}+O\left(\alpha^{3}, \alpha^{2} \frac{m_{e}}{m_{p}}\right) \\
\mathbf{K}_{A}=\varepsilon_{0} \alpha(0) \mathbf{B}_{0} \times \mathbf{E}_{0}
\end{gathered}
$$

Casimir momentum of $\mathrm{H}$ atom exists and slightly reduces the classical Abraham momentum

Casimir mass being equivalent to binding energy is same physics BaVT, Kawka, Rikken, submitted to EPJD 68 


\section{SUMMARY}

Casimir momentum in crossed $E, B$

- Classical Abraham force, linear in $E_{O}$ and $B_{0}$,

is observed for neutral atoms

(and for strong dielectrics)

- QED contribution by Feigel is not observed - UV divergencies disappear in mass renormalization. or cancel.

Need to go beyond multipole approximation

- Quantum vacuum contributes to

Abraham momentum in order $-(1 / 137)^{2}$

Will this be $-(Z / 137)^{2}$ for $Z>1$ ?? 
Magneto-Chiral Casimir momentum?

$$
\langle E \times B\rangle=g B_{0} \text { ? }
$$

- Classically no equivalent Abraham version in charge neutral systems

- g must be a pseudo scalar

$$
\rightarrow \text { medium must be chiral (on nanoscale) }
$$

- Describe chirality microscopically,

not phenomenologically using Lifshitz formula via « magneto-chiral « index of refraction $\left(\Delta n=g B_{0} \cdot k\right)$

- Would separate enantiomers using magnetic fields

= Pasteurs dream!

- Medium must have induced magnetic dipole since $\langle E \times H\rangle=0$ 
Pasteur's dream with a Casimir momentum $P=g B_{0}$ ?
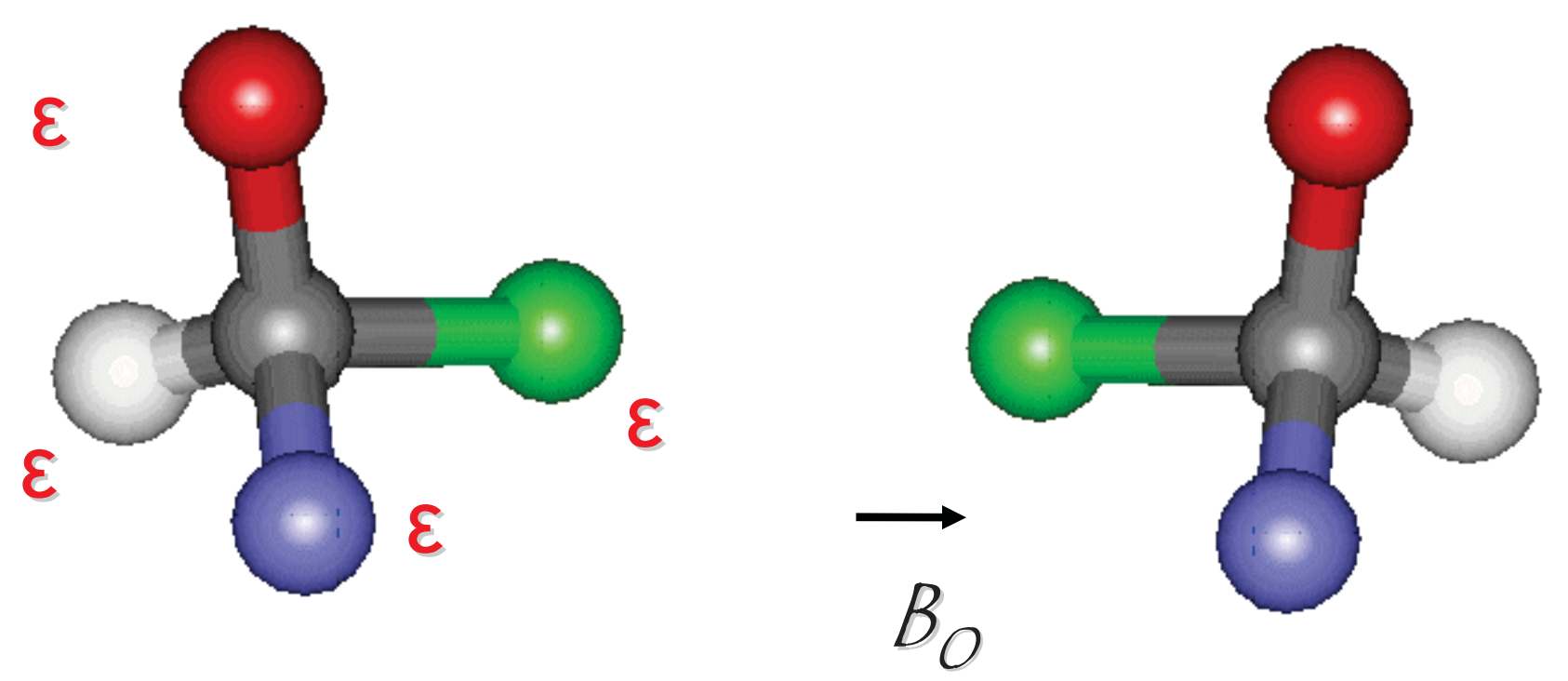

Chiral geometry with electric polarizabilities with Zeeman splitting Pinheiro and BAvT 69

$$
\alpha(\omega, \sigma)=\frac{4 \pi \mathrm{c}_{0}^{2}}{\omega_{0}^{2}} \frac{\gamma}{\omega^{2}-\omega_{0}^{2}+i \sigma V B+i \gamma \omega_{0}}
$$

$\mathbf{B}=\mu_{0} \mathbf{H} \Rightarrow\left\langle 0\left|\int d \mathbf{r} \mathbf{E} \times \mathbf{B}\right| 0\right\rangle \propto\left\langle 0\left|\int d \mathbf{r} \mathbf{E} \times \mathbf{H}\right| 0\right\rangle=0$ 
A Casimir momentum $P=g B_{0}$ ? Pasteur's dream!
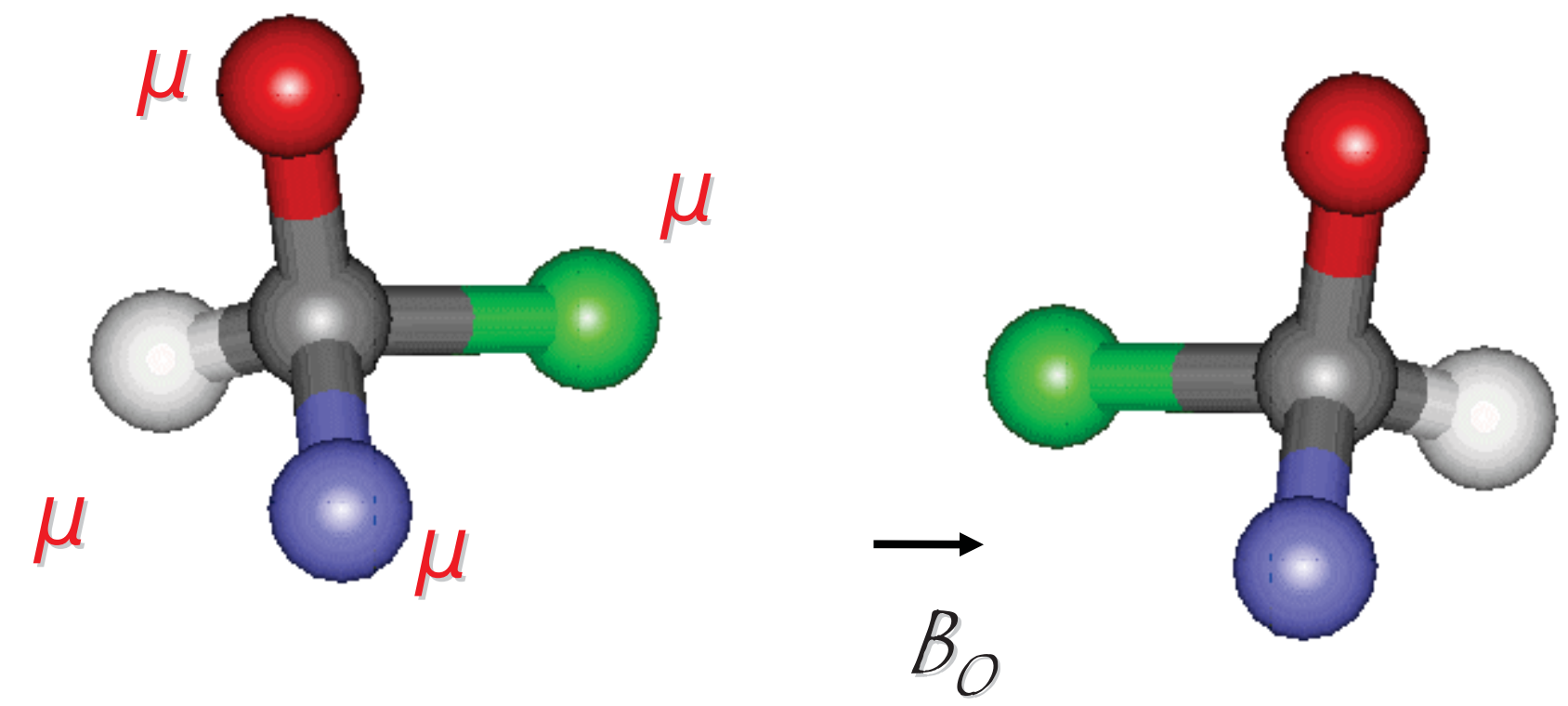

Chiral geometry with magnetic polarizabilities with Zeeman splitting

$$
\chi(\omega, \sigma)=\chi(0) \frac{\omega_{0}^{2}}{\omega^{2}-\omega_{0}^{2}+i \sigma V B+i \gamma \omega}
$$

$\left\langle 0\left|\int d \mathbf{r} \mathbf{E} \times \mathbf{H}\right| 0\right\rangle=0$

$\left\langle 0\left|\int d \mathbf{r} \mathbf{E} \times \mathbf{B}\right| 0\right\rangle=g \mathbf{B}_{0}$

$$
g=\left(\frac{4 \hbar c}{3 e \mu_{0}}\right)\left((\chi(0)) \mu_{0}\right)^{5}\left(\frac{1.4 \times 10^{4}}{L^{14}}\right)
$$

Na Tetraeder $L=10 \mathrm{~nm} \rightarrow \mathrm{g} / \mathrm{m}=1 \mathrm{~nm} / \mathrm{sec} / T$ Babington, BaVT, 201170 


\section{Very nonexhaustive bibliography}

See: James F. Babb (Harvard) : https://www.cfa.harvard.edu/ babb/casimir-bib.html

1. See M.L. Shih and P.W. Milonni, Am. J. Phys. 59, 684 (1991).

2. O. Stern and A. Einstein, Ann. Physik 40, 551 (1913).

3. For a simplified derivation see Appendix B of P.W. Milonni,

The Quantum Vacuum, an introduction to Quantum Electrodynamics,

Academic Press (San Diego 1994)

4. F. London, Z. Phys. 63, 245 (1930).

5. See e.g. N.D. Birrel and P.C.W. Davies, Quantum Fields in Curved Spaces

(Cambridge, 1984)

6. H. B. G. Casimir, and D. Polder, Physical Review, Vol. 73 (4), 360 (1948).

7. W.E. Lamb and R.C Retherford, Phys. Rev.72 (3): 241 (1947).

8. H. Bethe, Phys. Rev. 72, 339 (1947).

9. J. Schwinger, Phys. Rev. 73, 416L (1948).

10. H. B. G. Casimir, Proceedings of the Royal Netherlands Academy of

Arts and Sciences, 51, 793-795 (1948).

11. E. M. Lifshitz, Soviet Phys. JETP, 2, 73 (1956).

12. I.E.Dzyaloshinskii, IE.M. Lifshitz, L.P. Pitaevskii,,Sov.Phys. Uspekhi 4 ;153 (1961).

13. M.J. Sparnaay, Nature 180 (4581): 334; Physica 24 (6-10): 751 (1958).

14. S.K. Lamoreaux, Phys.Rev.Lett.78, 5 (1997).

15. U.Mohideen, A. Roy, Phys. Rev. Lett. 81 (21): 4549 (1998). 
16. T. Ederth, Phys. A 62, 062104 (2000).

17. H. G. B. Casimir, Physics 19, 846 (1956).

18. T. H. Boyer, Phys. Rev. 174, 1764 (1968).

19. S. W. Hawking, Nature 248 (5443): 30.

20. W.G. Unruh, Phys. Rev. D 14 (4): 870 (1976), . (1974).

21. S A Fulling and P C W Davies Proc. R. Soc. A 348393 (1976).

22. A. Chodos, R.L. Jaffe, etal, Phys. Rev. D 9, 3471 (1974).

23. S. Weinberg, Rev. Mod. Phys. 61, 1-23 (1989).

24. M.-T. Jaekel, S. Reynaud, J. Phys. I France 31093 (1993).

25. J. Schwinger, Proc. Natl. Acad. Sci. USA 89, 4091 (1992).

26. C. Eberlein, Phys. Rev. Lett. 76, 3842-3845 (1996)

27. L. S. Levitov ,Europhys. Lett. 84991989.

28. J. B. Pendry, J. Phys.:Cond. Matt. 9, 10301 (1997).

29. A. Feigel, Phys. Rev. Lett. 93, 268904 (2004).

30. O. Kenneth and I. Klich, Phys. Rev. Lett. 97160401 (2006)

31. A.A. Feiler etal, Langmuir, 24 (6), 2274 (2008).

32. J.N. Munday, F. Capasso, V.A. Parsegian, Nature 457, 7226 (2006).

33. K. A. Milton, S.A. Fulling etal, J.Phys.A41:164052 (2008). 
34. A. Lambrecht, P.A. Maia-Neto, and S. Reynaud, NJP 8, 243 (2006);

35. A.Lambrecht, A. Canaguier-Durand, R. Guérout and S. Reynaud, Lecture Notes in Physics, 834 (2011).

36. A. Canaguier-Durand, P. A. Maia Neto, A. Lambrecht, and S. Reynaud Phys. Rev. A 82, 012511 (2010)

37. S Reynaud, P.A.M. Neto P. A. M., A. Lambrecht A , J. Phys. A: Math. Theor. 41164004 (2008).

38. D. A. R. Dalvit, P. A. Maia Neto, A. Lambrecht, S. Reynaud, Phys. A: Math. Theor. 41, 164028 (2008).

37. M. Bordag, V. Nikolaev, J. Phys. A. Math. Theor. 41, 164002 (2008).

38. C.M. Wilson etal, Nature 479, 376 (2011).

39. A. O. Sushkov, W. J. Kim, D. A. R. Dalvit \& S. K. Lamoreaux

Nature Physics 7, 230 (2011)

40. K.A. Milton, The Casimir effect, World Scientific, Singapore, 2001

41. E.G. Adelberger, B.R. Heckel, A.E. Nelson, Ann.Rev. Nud. Part. Science Vol. 53: 77-1217 (2003)

42. D.J. Kapner etal, Rev. Lett. 98, 021101 (2007).

43. A. O. Sushkov, W. J. Kim, D. A. R. Dalvit \& S. K. Lamoreaux, Phys. Rev. Lett. 107, 171101 (2011).

44. P.W. Milonni, The Quantum Vacuum, an introduction to Quantum Electrodynamics, Academic Press (San Diego 1994). 
45. B.A. van Tiggelen, in: Atomic Matter Waves, edited by F. David, Ch. Westbrook, and R. Kaiser (Kluwer, Dordrecht, 2000)

46. I. Brevik, V.N. Marachevsky, K.A. Milton, Phys. Rev. Lett 82, 3948 - 3951 (1999)

47. S. Kawka, Ph.D. thesis, Université de Grenoble 1 (2010).

48. H. Gies and K. Klingmüller, Phys. Rev. Lett. 96, 220401 (2006).

49. A. Dupays, C. Rizzo, D. Bakalov, G.F. Bignami, EPL 82, 69002 (2008).

50. T.G. Philbin and U. Leonhardt, New ). Phys. 11, 033035 (2009).

51. J. Pendry, NJP 12, 033028 (2010).

52. J.S. Hoye and I. Brevik, EPL 91, 60003 (2010).

53. A.I. Volokitin and B.N.J. Persson, New J. Phys. 13 O68001(2011);

54. T.G. Philbin and U. Leonhardt, New ). Phys. 13068002 ( 2011).

55. G. Barton, New J. Phys. 12113044 (2010); New J. Phys. 12113045 (2010).

56. G. L. J. A. Rikken and C. Rizzo, Phys. Rev. A 63, 012107 (2003).

57. B.A. van Tiggelen and G.L.J.A. Rikken Phys. Rev. Lett. 100, 248902 (2008).

58. I. Brevik, Phys. Rep. 52, 133 (1979).

59. D.F. Nelson, Phys. Rev. A 44, 3985 (1991).

60. R. Peierls, Proc. Roy. Soc. A347 475.(1976).

61. G.B. Walker and G. Walker, Nature 263, 401 (1976); Nature 265, 324 (1977).

62. S.M. Barnett, Phys. Rev. Let. 104, 070401 (2010),;

S.M. Barnett and R. Loudon, Phil. Trans. R. Soc. A 368, 927 (2010)

63. A. Rizzo and S. Coriani, J. Chem. Phys. 119, 11064 (2003);

A. Rizzo, D. Shcherbin and K. Ruud, Can. J. Chem. 87:1352-1361 (2009). 
64. J. Babington and B.A. van Tiggelen, Eur. Phys. J. D 65, 367-372 (2011). 65. B.A. van Tiggelen, Eur. Phys. J. D 47, 261-269 (2008).

66. S. Kawka and B.A van Tiggelen, EPL 89, 11002 (2010);

$$
\text { erratum EPL 98, } 29904 \text { (2012). }
$$

67. G.L. J. A. Rikken, B. A. van Tiggelen ,Phys. Rev. Lett. 107, 170401 (2011) . 68. B.A. van Tiggelen, S. Kawka, G. L. J. A. Rikken, submitted to Eur. Phys. J. D 69. F. Pinheiro and B.A. van Tiggelen, Phys. Rev. E 66, 016607 (2002). 70. J. Babington, B. A. van Tiggelen, EPL 93, 41002 (2011)

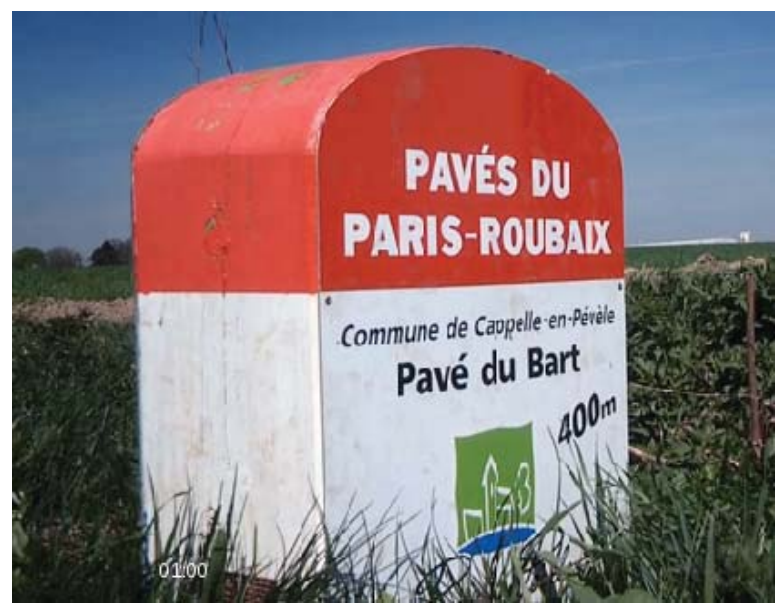

Thank you! 